\title{
MIMO-Aided Near-Capacity Turbo Transceivers: Taxonomy and Performance versus Complexity
}

\author{
Shinya Sugiura, Member, IEEE, Sheng Chen, Fellow, IEEE, and Lajos Hanzo, Fellow, IEEE
}

\begin{abstract}
In this treatise, we firstly review the associated Multiple-Input Multiple-Output (MIMO) system theory and review the family of hard-decision and soft-decision based detection algorithms in the context of Spatial Division Multiplexing (SDM) systems. Our discussions culminate in the introduction of a range of powerful novel MIMO detectors, such as for example Markov Chain assisted Minimum Bit-Error Rate (MC-MBER) detectors, which are capable of reliably operating in the challenging high-importance rank-deficient scenarios, where there are more transmitters than receivers and hence the resultant channelmatrix becomes non-invertible. As a result, conventional detectors would exhibit a high residual error floor. We then invoke the SoftInput Soft-Output (SISO) MIMO detectors for creating turbodetected two- or three-stage concatenated SDM schemes and investigate their attainable performance in the light of their computational complexity. Finally, we introduce the powerful design tools of EXtrinsic Information Transfer (EXIT)-charts and characterize the achievable performance of the diverse nearcapacity SISO detectors with the aid of EXIT charts.
\end{abstract}

Index Terms-EXIT chart, iterative detection, MIMO, multiuser detection, turbo coding, wireless communication.

\section{INTRODUCTION}

$\mathbf{M}$ ULTI-User Detectors (MUDs) were originally developed for the Code-Division Multiple Access (CDMA) uplink [1], where the direct-sequence spread symbols transmitted from the users supported are jointly estimated at the Base Station (BS), in order to minimize the associated MultipleAccess Interference (MAI), while exploiting the BS's knowledge of both the spreading sequences and of the supported users' Channel Impulse Responses (CIRs) [2]. However, as a benefit of the system model similarity between the CDMA uplink and the Space Division Multiplexing (SDM)-aided uplink [3], the family of MUDs developed for CDMA uplinks may be readily applied to Spatial Division Multiplexing (SDM) systems.

Figure 1 shows the classification of Multiple-Input MultipleOutput (MIMO) detectors. The class of linear MIMO detectors incorporated the classic Zero-Forcing (ZF) [4] and Minimum Mean Square Error (MMSE) detectors [5,6], as well as the

Manuscript received 10 October 2010; revised 22 January 2011. The financial support of the RC-UK under the auspices of the India-UK Advanced Technology Centre (IU-ATC) and the China-UK SCience Bridge in $4 \mathrm{G}$ Wireless Communications is gratefully acknowledged. The research leading to these results has also received funding from the European Union's Seventh Framework Programme ([FP7/2007-2013]) under grant agreement no [214625].

S. Sugiura is with the Toyota Central R\&D Laboratories, Inc., Nagakute, Aichi, 480-1192, Japan (e-mail: sugiura@ieee.org).

S. Chen and L. Hanzo are with the School of Electronics and Computer Science, University of Southampton, Southampton, SO17 1BJ, UK (e-mail: $\{$ sqc,lh\}@ecs.soton.ac.uk).

Digital Object Identifier 10.1109/SURV.2011.032511.00136 recently-proposed Minimum Bit-Error Rate (MBER) detector [7-10]. The linear MIMO detectors typically rely on adaptive MUD coefficient adjustment algorithms, such as the Least Mean Square (LMS) and Recursive Least Square (RLS) algorithms [11], which may be invoked both in the context of the classic MMSE criterion as well as in the recent Least Bit-Error Ratio (LBER) algorithm [12] relying on the MBER criterion. By contrast, the class of non-linear MIMO detectors enables us to achieve a higher BER performance than those of the linear detectors. The Maximum-Likelihood (ML) detector is capable of attaining the optimal performance in an uncoded scenario, which is achieved however at the cost of a prohibitively high complexity. In order to reduce the ML detector's complexity, the tree search-based Sphere Detector (SD) [13] was proposed for achieving a near-optimal BER performance by approximating the exhaustive ML search. Furthermore, the family of guided random detection algorithms, such as Genetic Algorithm (GA) [1, 14-16], Ant-Colony Optimization (ACO) [1719], Particle Swarm Optimization (PSO) [20-22] and Markov Chain Monte Carlo (MCMC) algorithms [23-25], was proposed for reduced-complexity near-optimum operation.

Moreover, since the invention of turbo coding [26], there has been a significant interest in iterative detection, where the receiver is constituted by multiple Soft-Input Soft-Output (SISO) decoders and each decoder iteratively exchanges extrinsic information gleaned from the other components [27]. During the iterative detection process, each SISO decoder is required to output soft information of the estimated bits. To this end, the diverse hard-decision MIMO detectors were extended to the SISO-assisted detectors, which are for example the SISO-MMSE [28,29], the SISO-MBER [30,31], the SISOMaximum A Posteriori (MAP) [5], the SISO-MCMC [32], the SISO-ACO [33] and the SISO-SD [34-36] detectors.

In this treatise, we introduce diverse hard-decision and soft-decision detectors in the context of SDM systems, while explicitly characterizing their fundamental complexity versus performance tradeoffs. The remainder of this chapter is organized as follows. Section II describes the system model of the SDM scheme considered in this chapter and guarantees the channel capacity. In Section III, a class of hard-decision MIMO detectors is introduced, including the recent Markov Chain MBER (MC-MBER) detector [37,38]. Furthermore, Section IV discusses the family of soft-decision detectors, in order to facilitate iterative detection. Section V analyzes the achievable performance with the aid of EXIT charts and quantifies the computational complexity of the SISO detectors. Finally, we summarize our findings and conclude in Section VI. 
This article has been accepted for inclusion in a future issue of this journal. Content is final as presented, with the exception of pagination.

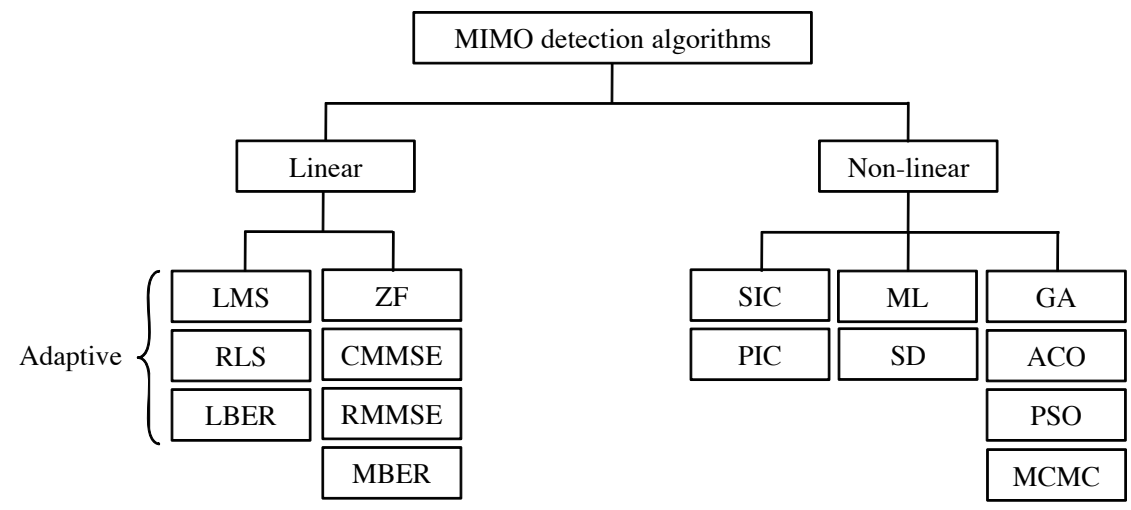

Fig. 1. Classification of MIMO detectors.

\section{SYSTEM OVERVIEW}

In this chapter, we consider the $(M \times N)$-element SDM system, which is portrayed in Fig. 2 and relies on $M$ transmit Antenna Elements (AEs) and $N$ receive AEs, while communicating over frequency-flat Rayleigh fading channels. Here, we employ the block-based system model of $\boldsymbol{Y}=\boldsymbol{H} \boldsymbol{S}+\boldsymbol{V}$, where the channel components $\boldsymbol{H} \in \mathcal{C}^{N \times M}$ and the noise components $\boldsymbol{V} \in \mathcal{C}^{N \times 1}$ follow zero-mean complex-valued Gaussian variables having variances of unity and of $N_{0}$, respectively. At the transmitter of Fig. $2, B=M \cdot \log _{2} \mathcal{L}$ information bits are mapped to $M$ number of $\mathcal{L}$-PSK/QAM substreams $\boldsymbol{S}=\left[s_{1}, s_{2}, \cdots, s_{M}\right]^{\mathrm{T}}$. Then, the $m$ th symbol $s_{m}$ is transmitted from the $m$ th transmit AE simultaneously with the other AEs' symbols. By contrast, the receiver has to decompose the $M$ multiplexed substreams, based on the received signals $\boldsymbol{Y}$, the estimated channel components $\boldsymbol{H}$ as well as the noise variance $N_{0}$.

\section{A. Channel Capacity}

\section{- CCMC capacity}

According to Telatar's tutorial paper [39], the ergodic capacity of the SDM system is formulated as $C_{\mathrm{CCMC}}=$ $E\left[\log _{2} \operatorname{det}\left(\boldsymbol{I}+\boldsymbol{H} \boldsymbol{H}^{\mathrm{H}} / N_{0}\right)\right]$. This ergodic capacity is also referred to as the Continuous-input Continuousoutput Memoryless Channel (CCMC) capacity, which is evaluated based on the effect of the symbol power and the bandwidth, but without taking into account the specific constellation size employed.

\section{- DCMC capacity}

In contrast to the above-mentioned CCMC capacity, the Discrete-input Continuous-output Memoryless Channel (DCMC) capacity was defined in [40], which serves as a more practical capacity bound than the CCMC capacity since it considers the effects of the specific signaling scheme employed. Hence, the DCMC capacity characterizes the maximum throughput limit in the high Signal-to-Noise Ratio (SNR) region, where the DCMC capacity curve saturates.

Given the $l$ th symbol $\boldsymbol{S}^{(l)}$ from the set of $N_{\mathrm{b}}=\mathcal{L}^{M}$ legitimate symbols $\left(l=1, \cdots, N_{\mathrm{b}}\right)$, the conditional
TABLE I

SYSTEM PARAMETERS OF THE UNCODED SDM SCHEME OF FIG. 3

\begin{tabular}{lr}
\hline \hline Number of transmit antennas & $M=2,3,4$ \\
Number of receive antennas & $N=3$ \\
Modulation & $\mathcal{L}-\mathrm{PSK} / \mathrm{QAM}$ \\
Channels & Frequency-flat Rayleigh fading \\
Channel's coherence-time & $\tau$ symbol durations \\
Transmission rate & $R_{\mathrm{SDM}}=M \cdot \log _{2} \mathcal{L}$ \\
\hline \hline
\end{tabular}

probability of the received signals $\boldsymbol{Y}$ is given by

$$
p\left(\boldsymbol{Y} \mid \boldsymbol{S}^{(l)}\right)=\frac{1}{\left(\pi N_{0}\right)^{N}} \exp \left(-\frac{\left\|\boldsymbol{Y}-\boldsymbol{H} \boldsymbol{S}^{(l)}\right\|^{2}}{N_{0}}\right) .
$$

Assuming that all the signals $\boldsymbol{S}^{(l)}$ are equiprobable, namely that we have $p\left(\boldsymbol{S}^{(1)}\right)=\cdots=p\left(\boldsymbol{S}^{\left(N_{\mathrm{b}}\right)}\right)=1 / N_{\mathrm{b}}$, the corresponding DCMC capacity $C_{\mathrm{DCMC}}$ is given by

$$
\begin{aligned}
C_{\mathrm{DCMC}} & =\left(\log _{2}\left(N_{\mathrm{b}}\right)-\frac{1}{N_{\mathrm{b}}}\right. \\
& \left.\times \sum_{l=1}^{N_{\mathrm{b}}} E\left\{\log _{2}\left[\sum_{k=1}^{N_{\mathrm{b}}} \exp \left(\boldsymbol{\Psi}_{k, l}\right) \mid \boldsymbol{S}^{(l)}\right]\right\}\right),
\end{aligned}
$$

with the relation $\boldsymbol{\Psi}_{k, l}=-\left\|\boldsymbol{H}\left(\boldsymbol{S}^{(k)}-\boldsymbol{S}^{(l)}\right)+\boldsymbol{V}\right\|^{2}+$ $\|\boldsymbol{V}\|^{2}$, where $E\left[\zeta \mid \boldsymbol{S}^{(l)}\right]$ indicates the expectation of $\zeta$ conditioned on $S^{(l)}$.

Fig. 3 shows the CCMC and DCMC capacity of the SDM scheme of Fig. 3, recorded for (a) BPSK and (b) QPSK modulation schemes, where the number of transmit AEs was varied from $M=2$ to $M=4$, while using $N=3$ receive AEs. It was found in both Figs. 3(a) and 3(b) that upon increasing the SNR value, the corresponding CCMC capacity increased infinitely, due to the assumption of Gaussian input signals. By contrast, the DCMC capacity was upperbounded by the corresponding system's achievable throughput of $R_{\mathrm{SDM}}=M \log _{2} \mathcal{L}$ at sufficiently high SNRs.

\section{MIMO DETECTORS FOR UnCODED SySTEMS}

In this section we describe the class of hard-decision detectors developed for the uncoded SDM scheme of Fig. 3 , where we commence with the family of linear detectors, which are classically referred to as spatial filtering schemes. In order to detect the $m$ th substream $s_{m}$ while suppressing 
This article has been accepted for inclusion in a future issue of this journal. Content is final as presented, with the exception of pagination.

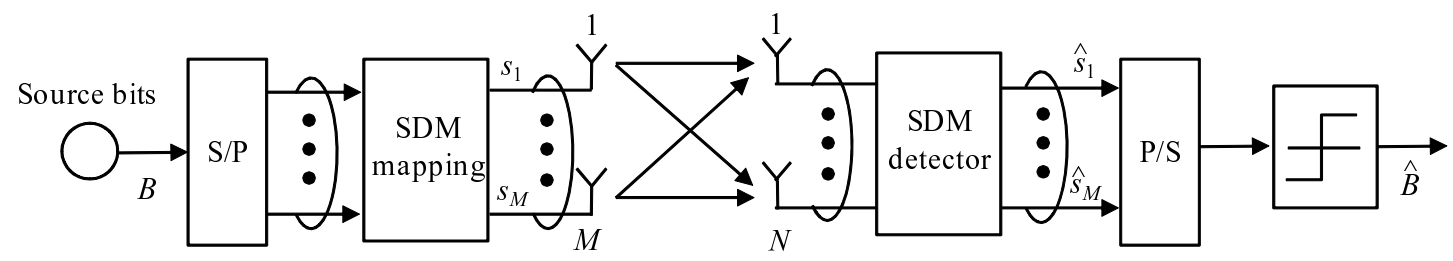

Fig. 2. Schematic of the uncoded $(M \times N)$-element SDM scheme.

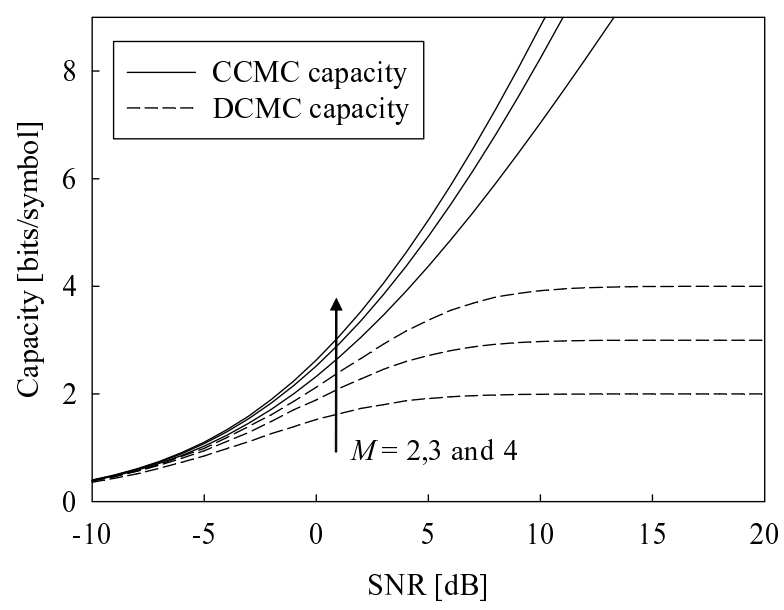

(a) BPSK

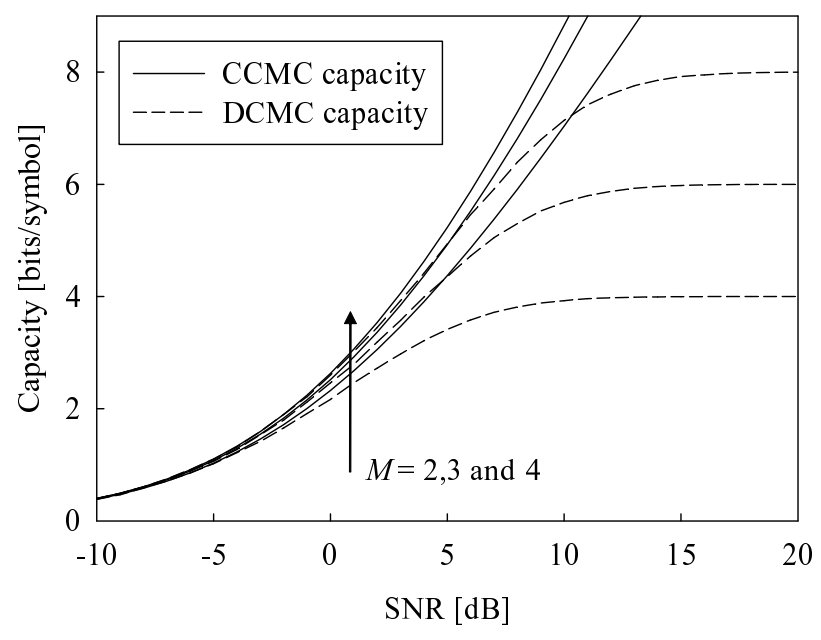

(b) QPSK

Fig. 3. CCMC and DCMC capacity curves of the SDM scheme obeying the architecture of Fig. 3, recorded for (a) BPSK and (b) QPSK modulation schemes, where the number of transmit antennas was varied from $M=2$ to $M=4$, while the number of receive antennas was $N=3$. All other system parameters were summarized in Table I.

the interfering $(M-1)$ substreams, a linear MIMO detector multiplies the received signals $\boldsymbol{Y}$ by the complex-valued weights $\boldsymbol{w}_{m}=\left[w_{1, m}, w_{2, m}, \cdots, w_{N, m}\right]^{\mathrm{T}}$, which is given by $\hat{s}_{m}=\boldsymbol{w}_{m}^{\mathrm{H}} \boldsymbol{Y}$. More specifically, the corresponding $M$ weight vectors are required for the sake of detecting all the $M$ substreams, which are represented by $\boldsymbol{W}=\left[\boldsymbol{w}_{1}, \boldsymbol{w}_{2}, \cdots, \boldsymbol{w}_{M}\right]$. Accordingly, the demodulated symbols $\hat{S}$ may be expressed in a vectorial form as $\hat{\boldsymbol{S}}=\left[\hat{s}_{1}, \hat{s}_{2}, \cdots, \hat{s}_{M}\right]^{\mathrm{T}}=\boldsymbol{W}^{\mathrm{H}} \boldsymbol{Y}$. In the class of linear detectors, once the complex-valued weights $\boldsymbol{W}$ are calculated, they can be reused for this filtering operation within the channel's coherence time $\tau$. Hence, the complexity imposed by the linear detectors is decreased upon increasing the value of $\tau$. Next, we will introduce the linear hard-decision ZF, MMSE and MBER detectors, followed by the non-linear ML, MCMC and MC-MBER detectors.

\section{A. Zero-Forcing Detector}

The classic ZF detector is the simplest linear detector, whose weights are given by the inverse of the channel matrix $\boldsymbol{H}^{-1}$. Hence, the estimated symbols $\hat{\boldsymbol{S}}$ are represented by $\hat{\boldsymbol{S}}=\boldsymbol{H}^{-1} \boldsymbol{Y}=\boldsymbol{S}+\boldsymbol{H}^{-1} \boldsymbol{V}$, where we have the corresponding inverse matrix $\boldsymbol{H}^{-1}$ [41]. ${ }^{1}$ Note that since the ZF detector does not take into account the effects of noise components $\boldsymbol{V}$, the

\footnotetext{
${ }^{1}$ Here, in order to calculate the inverse channel matrix of $\boldsymbol{H}$, we employed the approach of pseudo-inverse matrix, where we have $\boldsymbol{H}^{+}=$ $\left(\boldsymbol{H}^{H} \boldsymbol{H}\right)^{-1} \boldsymbol{H}^{H}$ for $M \leq N$ and $\boldsymbol{H}^{+}=\boldsymbol{H}^{H}\left(\boldsymbol{H} \boldsymbol{H}^{H}\right)^{-1}$ for $M>N$.
}

TABLE II

COMPUTATIONAL COMPLEXITY IMPOSED BY THE ZF DETECTOR

\begin{tabular}{|cc|r|r|}
\hline & & $\begin{array}{r}\text { Real-valued } \\
\text { multiplications }\end{array}$ & Inverse operation \\
\hline \hline$\left(\boldsymbol{H}^{\mathrm{H}} \boldsymbol{H}\right)^{-1}$ & $(M \leq N)$ & $4 M{ }^{2} N / \tau$ & $\mathcal{O}\left(M^{3}\right)$ \\
$\left(\boldsymbol{H} \boldsymbol{H}^{\mathrm{H}}\right)^{-1}$ & $(M>N)$ & $4 M N^{2} / \tau$ & $\mathcal{O}\left(N^{3}\right)$ \\
\hline$\bullet \times \boldsymbol{H}^{\mathrm{H}}$ & $(M \leq N)$ & $4 M^{2} N$ & - \\
$\boldsymbol{H}^{\mathrm{H}} \times \bullet$ & $(M>N)$ & $4 M N^{2}$ & - \\
\hline$\hat{\boldsymbol{S}=\bullet \times \boldsymbol{Y}}$ & $4 M N$ & - \\
\hline \hline Total & $(M \leq N)$ & $8 M^{2} N / \tau+4 M N$ & $\mathcal{O}\left(M^{3}\right)$ \\
& $(M>N)$ & $8 M N^{2} / \tau+4 M N$ & $\mathcal{O}\left(N^{3}\right)$ \\
\hline
\end{tabular}

resultant performance is typically degraded by the detrimental effects of noise enhancement.

\section{- Complexity analysis}

Here, we characterize the computational complexity imposed by the ZF detector in Table II, which is required to calculate the estimates $\hat{\boldsymbol{S}}$. Throughout this chapter, the complexity is evaluated in terms of the number of real-valued multiplications, noting that a single complexvalued multiplication is considered to be equivalent to four real-valued multiplications. Furthermore, the Cholesky decomposition of a Hermitian matrix having a dimension of $(C \times C)$ requires approximately $\mathcal{O}\left(C^{3}\right)$ real-valued multiplications. 
This article has been accepted for inclusion in a future issue of this journal. Content is final as presented, with the exception of pagination.

TABLE III

COMPUTATIONAL COMPLEXITY IMPOSED B Y THE MMSE DETECTOR

\begin{tabular}{|l|r|r|}
\hline & $\begin{array}{r}\text { Real-valued } \\
\text { multiplications }\end{array}$ & Inverse operation \\
\hline \hline $\boldsymbol{H H}^{\mathrm{H}}$ & $4 M N^{2} / \tau$ & - \\
\hline$\left(\bullet+\frac{N}{\rho} \boldsymbol{I}\right)^{-1}$ & - & $\mathcal{O}\left(N^{3}\right)$ \\
\hline$\bullet \times \boldsymbol{H}$ & $4 M N^{2} / \tau$ & - \\
\hline$\hat{\boldsymbol{S}=\bullet \times \boldsymbol{Y}}$ & $4 M N$ & - \\
\hline \hline Total & $8 M N^{2} / \tau+4 M N$ & $\mathcal{O}\left(N^{3}\right)$ \\
\hline
\end{tabular}

As mentioned above, the complex-valued weights $\boldsymbol{W}$ can be reused, while the channel matrix $\boldsymbol{H}$ remains constant, indicating that the associated computational complexity may be substantially reduced in slow-fading environments.

\section{B. Minimum Mean-Square Error Detector}

Similarly to the ZF detector of Section III-A, the MMSE detector belongs to the class of linear detectors. While the $\mathrm{ZF}$ detector suffers from effects of noise enhancement, the MMSE detector calculates the complex-valued weights by minimizing the MSE between the estimated symbol and the transmitted SDM symbol. Thus, the complex-valued weights are optimized in order to minimize the effects of noise enhancement.

More specifically, the minimization of the MSE between the $m$ th output $\hat{s}_{m}=\boldsymbol{w}_{m}^{\mathrm{H}} \boldsymbol{Y}$ and the $m$ th component of the symbol $s_{m}$ can be attained, according to the Wiener solution [11]: $\boldsymbol{w}_{m}=\left(E\left[\boldsymbol{Y} \boldsymbol{Y}^{\mathrm{H}}\right]\right)^{-1} E\left[\boldsymbol{Y} s_{m}^{*}\right]$. Furthermore, since we have $E\left[\boldsymbol{Y} \boldsymbol{Y}^{\mathrm{H}}\right]=\boldsymbol{H} E\left[\boldsymbol{S} \boldsymbol{S}^{\mathrm{H}}\right] \boldsymbol{H}^{\mathrm{H}}+E\left[\boldsymbol{V} \boldsymbol{V}^{\mathrm{H}}\right]=\boldsymbol{H} \boldsymbol{H}^{\mathrm{H}}+N_{0} \boldsymbol{I}$ and $E\left[\boldsymbol{Y} s_{m}^{*}\right]=\boldsymbol{H} E\left[\boldsymbol{S} s_{m}^{*}\right]=\boldsymbol{h}_{m}$, we arrive at $\boldsymbol{w}_{m}=$ $\left(\boldsymbol{H} \boldsymbol{H}^{\mathrm{H}}+N_{0} \boldsymbol{I}\right)^{-1} \boldsymbol{h}_{m}$. Finally, the complex-valued weights of the MMSE detector are given by $\boldsymbol{W}=\left[\boldsymbol{w}_{1}, \boldsymbol{w}_{2}, \cdots, \boldsymbol{w}_{M}\right]$ $=\left(\boldsymbol{H} \boldsymbol{H}^{\mathrm{H}}+N_{0} \boldsymbol{I}\right)^{-1} \boldsymbol{H}$. We note that the SDM system satisfying the relation of $M>N$ is referred to as a so-called rank-deficient scenario, where the attainable performance of the $\mathrm{ZF}$ and MMSE detectors is severely degraded by the residual interferences owing to the fact that $M$ streams have to be estimated with the aid of $N$ receive antennas.

\section{- Computational complexity}

The computational complexity imposed by the MMSE detector is characterized in Table III. Clearly, the MMSE detector exhibits a moderate computational complexity, which is comparable to that of the ZF detector. Additionally, periodical updates of the linear complex-valued weights $\boldsymbol{W}$ can be omitted when the channel matrix $\boldsymbol{H}$ remains constant, similarly to the above-mentioned $\mathrm{ZF}$ detector.

\section{Minimum Bit-Error Rate Detector}

Although the MMSE detection criterion is one of the most popular ones, minimizing the MSE does not necessarily guarantee the direct minimization of the system's BER. By contrast, the recently-proposed MBER detector [7-10] was designed to directly minimize the BER, hence it was shown to outperform the MMSE solution, provided that the detector's output is non-Gaussian.

Let us first define the $N_{\mathrm{b}}=\mathcal{L}^{M}$ number of legitimate transmitted sequences of $\boldsymbol{S}$ as $\boldsymbol{S}^{(l)}\left(l=1, \cdots, N_{\mathrm{b}}\right)$ in conjunction with the constellation order of $\mathcal{L}$. Then, considering the employment of BPSK modulation $(\mathcal{L}=2)$, we arrive at the error probability $P_{e}\left(\boldsymbol{w}_{m}\right)$ of the $m$ th substream signal $s_{m}$ in a closed form, which is represented as a function of the $m$ th complex weight. Having arrived at the exact BER expression, the MBER solution is given by $\boldsymbol{w}_{m}=\arg \min \boldsymbol{w}_{m} P_{e}\left(\boldsymbol{w}_{m}\right)$. Since in general $P_{e}\left(\boldsymbol{w}_{m}\right)$ is a nonlinear function of $\boldsymbol{w}_{m}$, therefore in general the error-probability optimization problem has to be solved iteratively, while for other linear detectors, such as the ZF and MMSE detectors, a closed-form solution can be derived. This suggests that the specific choice of the optimization method employed for finding the MBER weights affects both the computational complexity imposed and the achievable BER performance.

To this end, a number of algorithms, such as the steepest descent method [42], GAs [43] and PSO [44], were applied to the non-linear MBER detection problem. A promising approach is constituted by the Simplified Conjugate Gradient (SCG) method [42], which will be characterized in this treatise owing to its potential of delivering an efficient and stable solution for this problem. More specifically, the SCG method iteratively updates the tentative solution using the gradient vector of the error probability with respect to the $m$ th complex vector $\boldsymbol{w}_{m}$ as in [45]. This allows us to dispense with any perturbation operation, while generating the gradient, which reduces the associated complexity.

The above-mentioned MBER solution derived for BPSK modulation may be readily extended to QPSK, by conducting the error-probability minimization separately for both the realand imaginary-parts, which are denoted by $P_{e_{I}}$ and $P_{e_{Q}}$, respectively [46]. Furthermore, we may invoke the Minimum Symbol Error Rate (MSER) detector [47], where the MBER solution is applied to multilevel pulse-amplitude modulation, such as Quadrature Amplitude Modulation (QAM).

- Computational complexity

The total computational complexity imposed by the MBER detector is characterized in Table IV, where $N_{\mathrm{g}}$ is the number of iterations activated by the simplified conjugate algorithm used for finding the minimum of the BER versus MBER detector weight surface. It can be seen from Table IV that the MBER detector's complexity increases linearly with the number of legitimate sequences $N_{\mathrm{b}}$. Hence, upon increasing the multiplexing factor $M$, the associated complexity increases exponentially. For example, for a BPSK-modulated SDM system having $M=2$ transmit AEs, the value of $N_{\mathrm{b}}$ becomes as low as $N_{\mathrm{b}}=2^{2}=4$. On the other hand, the corresponding value of $N_{\mathrm{b}}$ for the case of a QPSKmodulated SDM system having $M=6$ transmit AEs becomes $N_{\mathrm{b}}=4^{6}=4096$, which represents a 1000 times higher value than that of the $M=2$ case employing BPSK. Naturally, the complex-valued weights have to be updated more frequently in fast-fading environments, which typically leads to a prohibitively-high complexity. To overcome this problem, a reduced-complexity version 
This article has been accepted for inclusion in a future issue of this journal. Content is final as presented, with the exception of pagination.

TABLE IV

COMPUTATIONAL COMPLEXITY IMPOSED BY THE MBER DETECTOR

\begin{tabular}{|l|r|r|}
\hline & Real-valued multiplications & exponential operation \\
\hline \hline $\begin{array}{l}\boldsymbol{H} \boldsymbol{S}^{(l)} \\
\left(l=1, \cdots, N_{\mathrm{b}}\right)\end{array}$ & $4 N_{\mathrm{b}} M N / \tau$ & - \\
\hline$\nabla P_{e}\left(\boldsymbol{w}_{m}\right)$ & $M\left(5 N_{\mathrm{b}} N+2 N_{\mathrm{b}}+1\right) \log _{2} \mathcal{L} / \tau$ & $N_{\mathrm{b}} M \log _{2} \mathcal{L} / \tau$ \\
\hline Weight update & $(18 M N+M) / \tau$ & - \\
\hline SCG loop & $\times N_{\mathrm{g}}$ & $\times N_{\mathrm{g}}$ \\
\hline All substreams & $\times M$ & $\times M$ \\
\hline$\hat{\boldsymbol{S}=\bullet \times \boldsymbol{Y}}$ & $4 M N$ & - \\
\hline \hline Total & $N_{\mathrm{g}} M^{2}\left[\left(4 N_{\mathrm{b}}+5 N_{\mathrm{b}} \log _{2} \mathcal{L}+18\right) N\right.$ & $N_{\mathrm{g}} N_{\mathrm{b}} M^{2} \log _{2} \mathcal{L} / \tau$ \\
& $\left.+\left(2 N_{\mathrm{b}}+1\right) \log _{2} \mathcal{L}+1\right] / \tau+4 M N$ & \\
\hline
\end{tabular}

of the MBER detector, a so-called Markov Chain-assisted MBER (MC-MBER) detector will be proposed in Section III-F.

Having reviewed the class of linear ZF, MMSE and MBER detectors. Let us now consider the family of non-linear detectors, namely the ML, the MCMC and the MC-MBER detectors.

\section{Maximum Likelihood Detector}

The above-mentioned linear detectors are based on the philosophy of spatial filtering, where each of the multiplexed signal streams is separated into unique spatial dimensions at the receiver. On the other hand, the ML detector has the capability of simultaneously identifying the spatially multiplexed signals by carrying out an exhaustive search over the legitimate signal space.

Let us first consider the classic MAP detection [34], which estimates the symbols $\hat{\boldsymbol{S}}$ by maximizing the a posteriori probability $P(\hat{\boldsymbol{S}} \mid \boldsymbol{Y})$, given an observation of $\boldsymbol{Y}$. Here particularly, the MAP criterion is given by $\hat{s}_{m}=\arg \max _{s_{m}} P\left(s_{m} \mid \boldsymbol{Y}\right)=$ $\arg \max _{s_{m}} \sum_{\forall \boldsymbol{S}^{(l)}: s_{m}^{(l)}=s_{m}} P\left(\boldsymbol{S}^{(l)} \mid \boldsymbol{Y}\right)$. Following from a Bayes' theorem, the a posteriori probability may be expressed as $P(\hat{\boldsymbol{S}} \mid \boldsymbol{Y})=p(\boldsymbol{Y} \mid \hat{\boldsymbol{S}}) P(\hat{\boldsymbol{S}}) / p(\boldsymbol{Y})$, where $p(\boldsymbol{Y})$ is constant for each candidate of the transmitted signals. Furthermore, assuming that the a priori probability $P(\hat{\boldsymbol{S}})$ is equal for all the estimated candidates, the MAP detector becomes equivalent to the ML detector, where the maximization of $P(\hat{\boldsymbol{S}} \mid \boldsymbol{Y})$ is equivalent to that of $p(\boldsymbol{Y} \mid \hat{\boldsymbol{S}})$. More specifically, the ML detector selects the estimates from the $N_{\mathrm{b}}=\mathcal{L}^{M}$ legitimate sequences so that the probability $p(\boldsymbol{Y} \mid \hat{\boldsymbol{S}})$ is maximized. Since the conditional probability of Eq. (1) is also referred to as the likelihood function, the ML solution is simplified to ${ }^{2}$ $\hat{\boldsymbol{S}}=\arg \min \boldsymbol{S}\|\boldsymbol{Y}-\boldsymbol{H} \boldsymbol{S}\|^{2}$.

- Computational complexity

The computational complexity imposed by ML detection per symbol vector $\boldsymbol{S}$ is shown in Table V. It was found that upon increasing the number of multiplexed streams $M$, the associated computational complexity increases exponentially, obeying the relationship of $N_{\mathrm{b}}=\mathcal{L}^{M}$. Additionally, since ML detection has to be conducted for each symbol interval, the associated complexity may become prohibitively high for a high value of $M$, although the ML detector achieves the optimum performance in an uncoded scenario.

\footnotetext{
${ }^{2}$ Note here the ML criterion comparing 2-dimensional Euclidean distances is possible only when each noise component follows a Gaussian distribution.
}

TABLE V

COMPUTATIONAL COMPLEXITY IMPOSED BY THE ML DETECTOR

\begin{tabular}{|c|r|}
\hline & $\begin{array}{r}\text { Real-valued } \\
\text { multiplications }\end{array}$ \\
\hline \hline $\boldsymbol{H} \boldsymbol{S}$ & $4 M N$ \\
\hline$\|\boldsymbol{Y}-\bullet\|^{2}$ & $4 M$ \\
\hline arg min & $\times N_{\mathrm{b}}$ \\
\hline \hline Total & $4 N_{\mathrm{b}}(M N+M)$ \\
\hline
\end{tabular}

As mentioned, the ML detector is optimal, when all the a priori probabilities are equal. However, in order to achieve the optimal performance for a channel-coded system, where additional apriori information may be invoked at the receiver, the MAP detector [34] achieves the optimal performance, since it exploits the a priori probability.

\section{E. Markov Chain Monte Carlo Detector}

As mentioned above, although the ML detector achieves the best attainable performance in an uncoded scenario, its complexity is high. To this end, the recent studies of Markov chain sampling have produced reduced-complexity solutions for wireless communication systems [23-25,32]. More specifically, the MCMC detector [23-25,32] constitutes an alternative sub-optimal non-linear detector, which is based on the efficient extraction of the statistical inferences with the aid of Markov chains [23-25].

More specifically, the MCMC algorithm is based on two different techniques, i.e. on the so-called Markov chain representation and on Monte Carlo integration. While the former is employed to find the most likely detection candidates according to the associated probability distributions, the latter is used to approximate the integral of interest on the basis of the detection candidates calculated by the Markov chain representation.

1) Markov chain representation: Several algorithms have been designed for finding the most likely decision candidate set with the aid of a Markov chain process [25]. In this treatise we employ the most popular so-called Gibbs-Sampler, which assists us in sampling the detection candidates set, with the aim of finding the most likely ones. Fig. 4 portrays a flowchart of the Gibbs-Sampler algorithm employed for the MCMC detector, where the algorithmic steps are as follows:

\section{1) Initialization:}

The initialization block of the Gibbs-Sampler of Fig. 
This article has been accepted for inclusion in a future issue of this journal. Content is final as presented, with the exception of pagination.

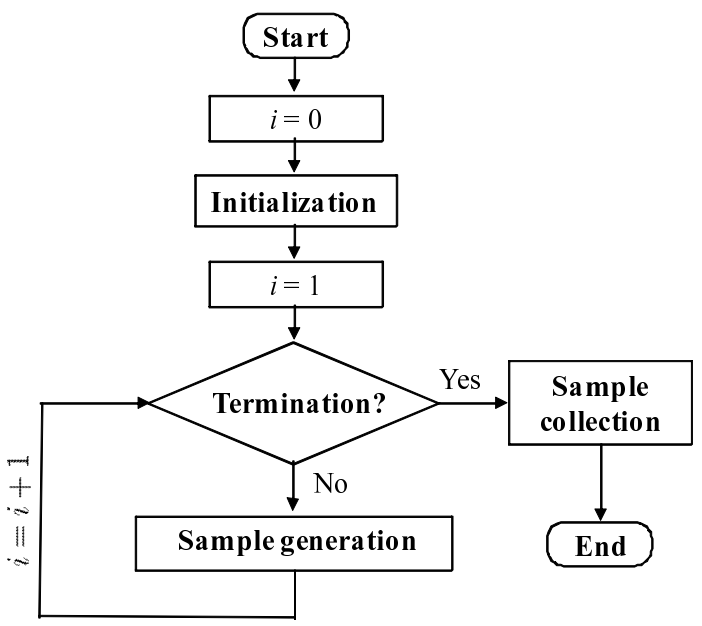

Fig. 4. A flowchart depicting the structure of Gibbs-Sampler employed for the MCMC detector.

4 randomly generates $B=M \log _{2} \mathcal{L}$ binary signals $\boldsymbol{B}^{(i=0)}=\left[b_{1}^{(0)}, \cdots, b_{B}^{(0)}\right]^{\mathrm{T}}$, which represents one of the $N_{\mathrm{b}}=\mathcal{L}^{M}$ legitimate signal sequences and the index $i$ indicates the number of loops of Fig. 4.

2) Sample generation:

In the sample generation block of Fig. 4, the binary signals $\boldsymbol{B}^{(i)}=\left[b_{1}^{(i)}, \cdots, b_{B}^{(i)}\right]^{\mathrm{T}}$ generated during the $i$ th loop are calculated based on the $(i-1)$ st binary signals $\boldsymbol{B}^{(i-1)}$, on the received signals $\boldsymbol{Y}^{(i)}$ and on the estimated channels $\boldsymbol{H}$. To be more specific, the $k$ th bit $b_{k}^{(i)}$ of the signals $\boldsymbol{B}^{(i)}$ in the $i$ th loop is generated from the conditional probability of $P\left(b_{k}^{(i)}=0 \mid \boldsymbol{Y}, \boldsymbol{B}_{-k}^{(i)}, \boldsymbol{L}_{1}^{\mathrm{pr}}\right)$, where we have $\boldsymbol{B}_{-k}^{(i)}=$ $\left[b_{1}^{(i)}, \cdots, b_{k-1}^{(i)}, b_{k+1}^{(i-1)}, \cdots, b_{B}^{(i-1)}\right]^{\mathrm{T}}$.

3) Sample collection:

This sample generation block is activated for $N_{\mathrm{MC}}$ iterations, thus a total of $N_{\mathrm{MC}}$ signals $\boldsymbol{B}^{(i)}(i=$ $\left.1, \cdots, N_{\mathrm{MC}}\right)$ are generated.

The above-mentioned Gibbs-Sampler of Fig. 4 samples a set of likely signals with the aid of a random variable so that the likelihood function is maximized as a result. Owing to the iterative process of Fig. 4, a chain of $N_{\mathrm{MC}}$ sampling processes are correlated since the initial condition of the $(i+1)$ st loop is the outcome of the $i$ th loop and hence all of the signals are originated from the initial conditions $\boldsymbol{B}^{(0)}$. Therefore, $N_{\mathrm{P}}$ parallel Gibbs-Samplers may be invoked to avoid the problem of having highly correlated successive Gibbs-Sampler solutions. The employment of this method results in an increased number of detection candidates $N_{\mathrm{MC}} N_{\mathrm{P}}$.

To elaborate a little further, Fig. 5 shows a simple example of the Gibbs-Sampler derived for the case of an 8-PAM SDM system having $M=3$ transmit AEs, where the corresponding number of legitimate sequences $N_{\mathrm{b}}$ was $N_{\mathrm{b}}=8^{3}=512$ and the SNR was set to $5 \mathrm{~dB}$. While Fig. 5(a) shows the legitimate signal space, Fig. 5(b) represents the reduced-size signal space. Here, the Gibbs-Sampler's parameters were given by $N_{\mathrm{MC}}=50$ and $N_{\mathrm{P}}=1$. Observe in Fig. $5(\mathrm{~b})$ that the sampled signals were distributed around the transmitted signal due to the criterion associated with the likelihood function, although this does not necessarily mean that the sampled signals include the transmitted signals. As a result of the Gibbs-Sampling operation, the signal space was reduced to $N_{\mathrm{b}}^{\prime}=34$, indicating that a $512 / 34=15.1$ times lower number of detection candidates were evaluated than the total number of legitimate sequences.

2) Monte Carlo integration: Having obtained a reduced number of $N_{\mathrm{MC}} N_{\mathrm{P}}$ decision candidates for signal set $\boldsymbol{B}^{(i)}\left(i=1, \cdots, N_{\mathrm{MC}}\right)$ with the aid of the Gibbs-Sampler of Fig. 4, we infer the estimate of the transmitted signals with the aid of a process referred to as Monte Carlo integration. In general, the process of Monte Carlo integration may be carried out by two different techniques, namely either by empirical averaging or by importance sampling steps. The corresponding estimates $E[\psi(x)]$ of $\psi(x)$ may be generated from the samples $\psi\left(x^{(m)}\right)\left(m=1, \cdots, N_{\mathrm{MC}}\right)$ as, respectively, [32] $E[\psi(x)]=\frac{1}{N_{\mathrm{MC}}} \sum_{m} \psi\left[x^{(m)}\right]$ (empirical average) and $E[\psi(x)]=\frac{\sum_{m} f\left[x^{(m)}\right] \psi\left[x^{(m)}\right]}{\sum_{m} f\left[x^{(m)}\right]}$ (importance sampling), where $f\left[x^{(m)}\right]$ represents a weighting function. Since the latter typically exhibits a better performance, we employ this technique in our investigations.

The decision concerning the $k$ th bit $b_{k}$ may be formulated by considering the extrinsic Log-Likelihood Ratio (LLR) value represented by $L_{1}^{\mathrm{e}}\left(b_{k}\right)=\ln \frac{P\left(b_{k}=0 \mid \boldsymbol{Y}, \boldsymbol{L}_{1}^{\mathrm{pr}}\right)}{P\left(b_{k}=1 \mid \boldsymbol{Y}, \boldsymbol{L}_{1}^{\mathrm{pr}}\right)}$. In order to estimate $L_{1}^{\mathrm{e}}\left(b_{k}\right)$ from a set of sampled signals, we first infer the probability $P\left(b_{k}=0 \mid \boldsymbol{Y}, \boldsymbol{L}_{1}^{\mathrm{pr}}\right)$ based on the importance sampling technique [32], which is then reformulated using Bayes rule. As a result, we have

$$
L_{1}^{\mathrm{po}}\left(b_{k}\right)=\underbrace{\ln \frac{\sum p\left(\boldsymbol{Y} \mid \boldsymbol{B}_{-k}^{(i)}, b_{k}=0\right) P\left(\boldsymbol{B}_{-k}^{(i)} \mid \boldsymbol{L}_{1}^{\mathrm{pr}}\right)}{\sum p\left(\boldsymbol{Y} \mid \boldsymbol{B}_{-k}^{(i)}, b_{k}=1\right) P\left(\boldsymbol{B}_{-k}^{(i)} \mid \boldsymbol{L}_{1}^{\mathrm{pr}}\right)}}_{L_{1}^{\mathrm{e}}\left(b_{k}\right)}+L_{1}^{\mathrm{pr}}\left(b_{k}\right),
$$

where $L_{1}^{\mathrm{po}}$ and $L_{1}^{\mathrm{pr}}$ represent the a posteriori and a priori LLR values, respectively [27]. While $p\left(\boldsymbol{Y} \mid \boldsymbol{B}_{-k}^{(i)}, b_{k}=0,1\right)$ is calculated during the Gibbs-Sampling process, $P\left(\boldsymbol{B}_{-k}^{(i)} \mid \boldsymbol{L}_{1}^{\mathrm{pr}}\right)$ is given by $1 / N_{\mathrm{b}}$ in the non-iterative hard-decision based scenario considering $\boldsymbol{L}_{1}^{\mathrm{pr}}=\left[L_{1}^{\mathrm{pr}}\left(b_{1}\right), \cdots, L_{1}^{\mathrm{pr}}\left(b_{B}\right)\right]^{\mathrm{T}}=$ $[0, \cdots, 0]^{\mathrm{T}}$.

Finally, by subjecting the a posteriori probability $L_{1}^{\mathrm{po}}\left(b_{k}\right)(k=1, \cdots, B)$ to the hard-decision operation, the output of the MCMC detector can be obtained.

- Computational complexity

Table VI shows the computational complexity imposed by the Gibbs-Sampler of Fig. 4, which is employed by the MCMC detector.

\section{F. Markov Chain Minimum Bit Error Rate Detector}

As mentioned in Section III-C, the family of MBER detectors $[7-10,12,30]$ was designed to directly minimize the BER, and hence it was shown to outperform the MMSE solution in the context of beamforming [7], Space-Time Equalization (STE) [9] and SDMA [8], supporting either BPSK or QPSK modulation schemes [12]. Furthermore, it was demonstrated that the MBER receiver has the capability of operating in rankdeficient scenarios, where the number of transmit antennas is 
This article has been accepted for inclusion in a future issue of this journal. Content is final as presented, with the exception of pagination.

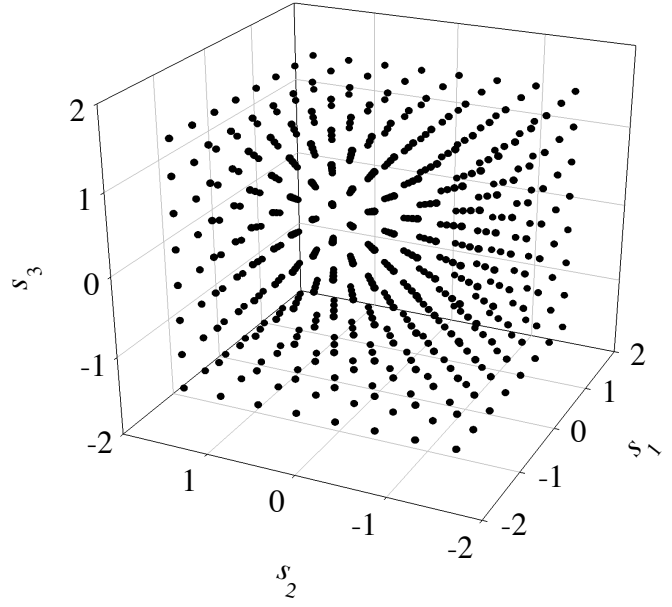

(a) Legitimate signal space

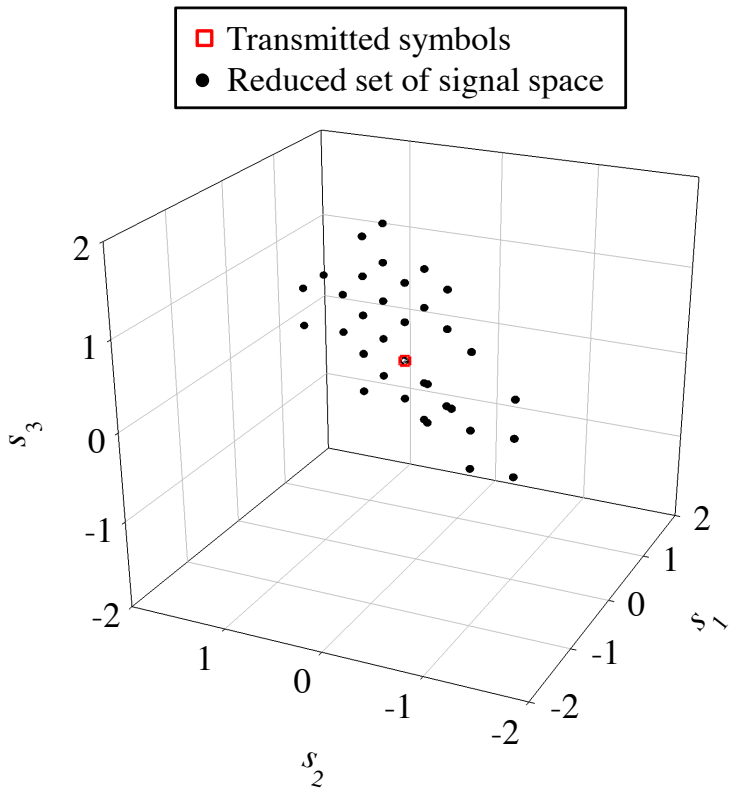

(b) Reduced-size signal space

Fig. 5. Example of the Gibbs-Sampling assisted signal space reduction for an 8-Pulse Amplitude Modulation (PAM) SDM system having $M=3$ transmit AEs. The number of legitimate signals $N_{\mathrm{b}}$ is given by $N_{\mathrm{b}}=8^{3}=256$, the resultant reduced-size signal space $N_{\mathrm{b}}^{\prime}$ was $N_{\mathrm{b}}^{\prime}=34$, where $N_{\mathrm{MC}}=50$ successive samples were generated according to the Gibbs-Sampler.

TABLE VI

COMPUTATIONAL COMPLEXITY IMPOSED BY THE GIBBS-SAMPLER EMPLOYED FOR THE MCMC DETECTOR

\begin{tabular}{|c|r|r|}
\hline & Real-valued multiplications & Exponential \\
\hline \hline$\left\|\boldsymbol{Y}-\boldsymbol{H} \boldsymbol{S}_{m \pm}^{(i)}\right\|^{2}$ & $\leq 4\left(1+N_{\mathrm{MC}} N_{\mathrm{P}} M \log _{2} \mathcal{L}\right)$ & \\
& $\times(M N+M)$ & \\
\hline$P\left(x_{m}^{(i)} \mid \boldsymbol{x}_{-m}^{(i)}, \boldsymbol{Y}\right)$ & $2 N_{\mathrm{MC}} N_{\mathrm{P}} M \log _{2} \mathcal{L}$ & $N_{\mathrm{MC}} N_{\mathrm{P}} M \log _{2} \mathcal{L}$ \\
\hline \hline Total & $\leq 4\left(1+N_{\mathrm{MC}} N_{\mathrm{P}} M \log _{2} \mathcal{L}\right)$ & $N_{\mathrm{MC}} N_{\mathrm{P}} M \log _{2} \mathcal{L}$ \\
& $\times(M N+M)+2 N_{\mathrm{MC}} N_{\mathrm{P}} M \log _{2} \mathcal{L}$ & \\
\hline
\end{tabular}

higher than the number of receive antennas. However, the high BER performance of the MBER scheme is achieved at the cost of a high computational complexity, which may become particularly challenging in rapidly fading propagation environments, requiring prompt MBER detector weight updates. To be more specific, the calculation of the MBER weight gradient imposes a prohibitively-high complexity, which increases with the number of transmit AEs $M$. To this end, we introduce a novel MCMC aided MBER (MC-MBER) algorithm for the sake of reducing the computational complexity of the conventional MBER algorithm without degrading its performance.

Fig. 6 shows the flowchart of the proposed MC-MBER detector, which is composed of two blocks, i.e. the Markov chain sampling block and the approximated MBER detector block. Firstly, in our MC-MBER detector, the Markov chain sampling is implemented to generate the most likely $N_{M C} N_{\mathrm{P}}$ number of signals $\boldsymbol{S}^{(i)}\left(i=1, \cdots, N_{M C} N_{\mathrm{P}}\right)$ that particularly contribute to the BER calculation of the MBER detector. Here, we also employ the Gibbs-Sampler for the Markov chain sampling, similarly to the MCMC detector. Having completed the generation of the Gibbs-Sampler's detection candidate set of $N_{M C} N_{\mathrm{P}}$ signals, only $N_{\mathrm{b}}^{\prime}<N_{\mathrm{b}}$ number of detection candidates $\boldsymbol{S}^{(i)}\left(i=1, \cdots, N_{b}^{\prime}\right)$ are retained from the GibbsSampler's solution set, also ensuring that the identical detec- tion candidates of the parallel Gibbs-Samplers are removed.

These $N_{\mathrm{b}}^{\prime}<N_{\mathrm{b}}$ detection candidates are then input to the approximate MBER detector of Fig. 6. More specifically, the detection candidates are used for calculating the gradient by replacing $N_{\mathrm{b}}$ number of legitimate sequences by a reduced set of $N_{\mathrm{b}}^{\prime}<N_{\mathrm{b}}$ signals. The underlying concept of this approximate MBER detector is that the unlikely signals, which are not sampled by the Gibbs-Sampler, do not substantially contribute the BER minimization. Typically, $N_{\mathrm{b}}^{\prime}$ becomes significantly lower than $N_{\mathrm{b}}=\mathcal{L}^{M}$, which is an explicit benefit of the rapid convergence of the Gibbs-Sampler of Fig. 4.

- Computational Complexity

The computational complexity imposed by the MCMBER detector is shown in Table VII, which is based on the analysis of the MBER detector detailed in Section III-C and on the MCMC detector discussed in Section III-E. The computational complexity of the MC-MBER detector, which is imposed by calculating the gradient of the BER with respect to the weights can be reduced by a factor of $N_{b} / N_{b}^{\prime}$ in comparison to that of the full-complexity MBER scheme, although the MC-MBER detector imposes the additional computation of the GibbsSampler based reduced set of $N_{b}^{\prime}<N_{b}$ signals. 
This article has been accepted for inclusion in a future issue of this journal. Content is final as presented, with the exception of pagination.

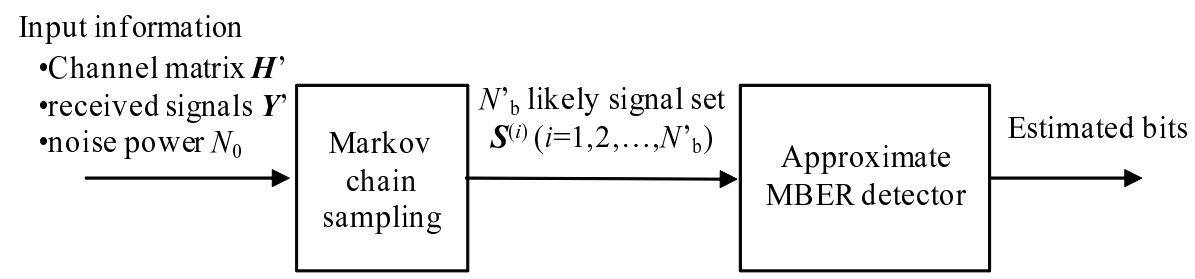

Fig. 6. A flowchart of the MC-MBER detector.

TABLE VII

COMPUTATIONAL COMPLEXITY FOR AN MC-MBER DETECTOR

\begin{tabular}{|l|r|r|}
\hline & $\begin{array}{r}\text { Real-valued } \\
\text { multiplications }\end{array}$ & $\begin{array}{r}\text { exponential } \\
\text { operation }\end{array}$ \\
\hline \hline Markov chain & $\leq 4\left(1+N_{\mathrm{MC}} N_{\mathrm{P}} M \log _{2} \mathcal{L}\right)$ & $N_{\mathrm{MC}} N_{\mathrm{P}} M \log _{2} \mathcal{L}$ \\
sampling & $\times(M N+M)+2 N_{\mathrm{MC}} N_{\mathrm{P}} M \log _{2} \mathcal{L}$ & \\
\hline Approximated & $4\left(N_{\mathrm{b}}^{\prime} \log _{2} \mathcal{L}+N_{\mathrm{b}}^{\prime} N_{\mathrm{g}}+3 N_{\mathrm{g}}\right) M N$ & $N_{\mathrm{g}} M N_{\mathrm{b}} \log _{2} \mathcal{L}$ \\
MBER detector & $+M\left\{\left(N_{\mathrm{b}}^{\prime} N+2 N_{\mathrm{b}}^{\prime}+1\right) \log _{2} \mathcal{L}+6 N+1\right\}$ & \\
\hline
\end{tabular}

\section{G. Center-Shifted Sphere Detector}

In this section, we introduce the center-shifted SD, which constitutes another computationally efficient solution to the ML detection. Note that while the above-mentioned MCMC detector approximate the ML detector with the aid of stochastic sampling, the SD aim for deterministic approximation of the ML detection.

More specifically, the ML solution of Section III-D may be rewritten by [48]

$$
\begin{aligned}
\hat{\boldsymbol{S}} & =\arg \min _{\boldsymbol{S} \in \boldsymbol{\chi}} J(\boldsymbol{S}) \\
& =\arg \min _{\boldsymbol{S} \in \boldsymbol{\chi}}\left(\boldsymbol{S}-\boldsymbol{X}_{c}\right)^{H}\left(\boldsymbol{H}^{H} \boldsymbol{H}+N_{0} \boldsymbol{I}\right)\left(\boldsymbol{S}-\boldsymbol{X}_{c}\right),
\end{aligned}
$$

where $\boldsymbol{X}_{c}$ represents the MMSE solution of $\boldsymbol{X}_{c}=$ $\left[x_{c, 1}, \cdots, x_{c, M}\right]=\left(\boldsymbol{H}^{H} \boldsymbol{H}+N_{0} \boldsymbol{I}\right)^{-1} \boldsymbol{H}^{H} \boldsymbol{Y}$ and $\boldsymbol{\chi}$ denotes the legitimate signal space of $\boldsymbol{S}$. To be more specific, by exploiting the characteristics of the upper triangle matrix $\boldsymbol{U}$, which is defined as $\boldsymbol{U}^{H} \boldsymbol{U}=\boldsymbol{H}^{H} \boldsymbol{H}+N_{0} \boldsymbol{I}$, the cost function $J(\boldsymbol{S})$ of Eq. (3) may be formulated as

$$
\begin{aligned}
J(\boldsymbol{S}) & =\left(\boldsymbol{S}-\boldsymbol{X}_{c}\right)^{H} \boldsymbol{U}^{H} \boldsymbol{U}\left(\boldsymbol{S}-\boldsymbol{X}_{c}\right) \\
& =\sum_{i}^{M} \underbrace{\left|\sum_{j}^{M} u_{i, j}\left(s_{j}-x_{c, j}\right)\right|^{2}}_{\phi_{i}\left(\boldsymbol{S}_{i}\right)},
\end{aligned}
$$

where $u_{i, j}$ represents the $i$-th row and the $j$ th column element of $\boldsymbol{U}$, while we have $\boldsymbol{S}_{i}=\left[s_{i}, \cdots, s_{M}\right]$. Furthermore, $\phi_{i}\left(\boldsymbol{S}_{i}\right)$ may be elaborated as [48]

$$
\begin{aligned}
\phi_{i}\left(\boldsymbol{S}_{i}\right) & =\left|\sum_{j}^{M} u_{i, j}\left(s_{j}-x_{c, j}\right)\right|^{2} \\
& =\left|u_{i, i}\left(s_{i}-x_{c, i}\right)+\sum_{j=i+1}^{M} u_{i, j}\left(s_{j}-x_{c, j}\right)\right|^{2} .
\end{aligned}
$$

Here, let us define a cumulative sub-cost function $J_{i}\left(\boldsymbol{S}_{i}\right)$ in a recursive manner as

$$
J_{i}\left(\boldsymbol{S}_{i}\right)=J_{i+1}\left(\boldsymbol{S}_{i+1}\right)+\phi_{i}\left(\boldsymbol{S}_{i}\right)(i=M-1, \cdots, 1),
$$

where we have $J_{M}\left(\boldsymbol{S}_{M}\right)=\phi_{M}\left(\boldsymbol{S}_{M}\right)=$ $\left|u_{M, M}-\left(s_{M}-x_{c, M}\right)\right|^{2}$. Then, we have the following properties of $J(\boldsymbol{S})=J_{1}\left(\boldsymbol{S}_{1}\right)>\cdots>J_{M}\left(\boldsymbol{S}_{M}\right)>0$ and $J_{i}\left(\boldsymbol{S}_{i}\right)=J_{i}\left(\left\{s_{j}\right\}, j=i, \cdots, M\right)$ for all the possible signals of $\boldsymbol{S} \in \mathcal{L}^{M}$. Hence, Eqs. (4) and (5) enable us to facilitate the low-complexity tree search algorithm, which is capable of avoiding the exhaustive search. The detailed implementation can be found in [48].

We note that the complexity of the SD may be further reduced by choosing an appropriate value for the maximum number of best MIMO-symbol candidates $K$ retained at each search level in the so-called $K$-best SD [49].

\section{H. Performance Results}

In this section, we present the performance results characterizing diverse MIMO detectors in the context of SDM systems obeying the architecture of Fig. 2 employing $M=2,3$ and 4 transmit AEs and $N=3$ receive AEs. Here, we considered the employment of BPSK and QPSK modulation.

Firstly, Fig. 7 compares the achievable BER performance of the linear detectors, namely the ZF detector of Section III-A, the MMSE detector of Section III-B and the MBER detector of Section III-C. Here, we considered BPSK modulation. It was found that the achievable BER performance of the MMSE detector was better than that of the $\mathrm{ZF}$ detector, since the MMSE detector optimizes its complex-valued weights so as to minimize the effects of AWGN. By contrast, both the ZF and MMSE detectors exhibited error floors in the rank-deficient scenario of $M=4$. Furthermore, observe in Fig. 7 that unlike the $\mathrm{ZF}$ and MMSE detectors the MBER detector did not show any error floors. This is, because the MBER detector is more robust to the rank-deficient scenario than the other two linear detectors, which is an explicit benefit of the direct BER minimization.

Next, we characterize the performance results of the nonlinear detectors. Fig. 8 shows the achievable BER of the optimal ML detector seen in Section III-D. Observe in Fig. 
This article has been accepted for inclusion in a future issue of this journal. Content is final as presented, with the exception of pagination.

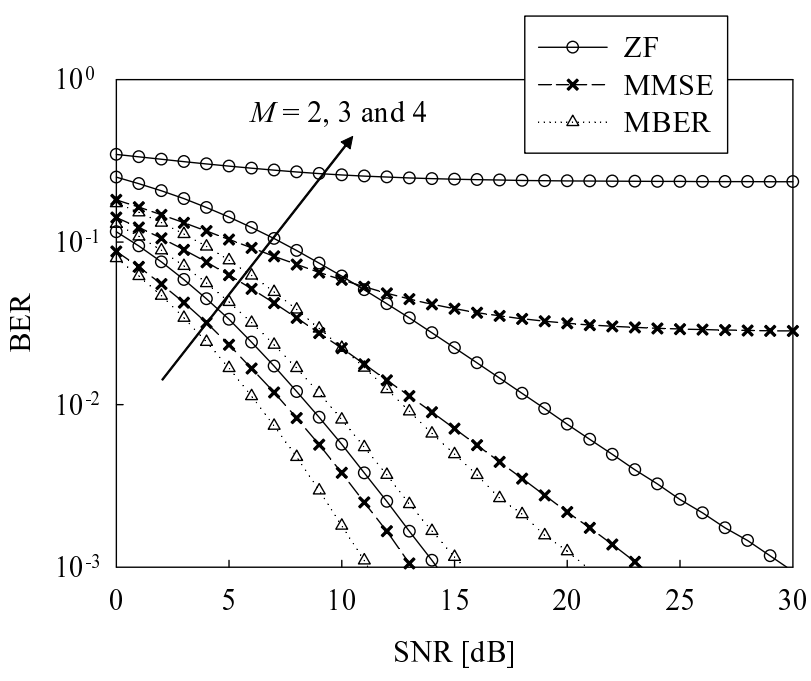

Fig. 7. Achievable BER performance of the uncoded SDM scheme obeying the architecture of Fig. 2 and employing the ZF, the MMSE and the MBER detectors, where the number of transmit antennas was varied from $M=2$ to $M=4$, while the number of receive antennas was maintained to be $N=3$. All other system parameters were summarized in Table I.

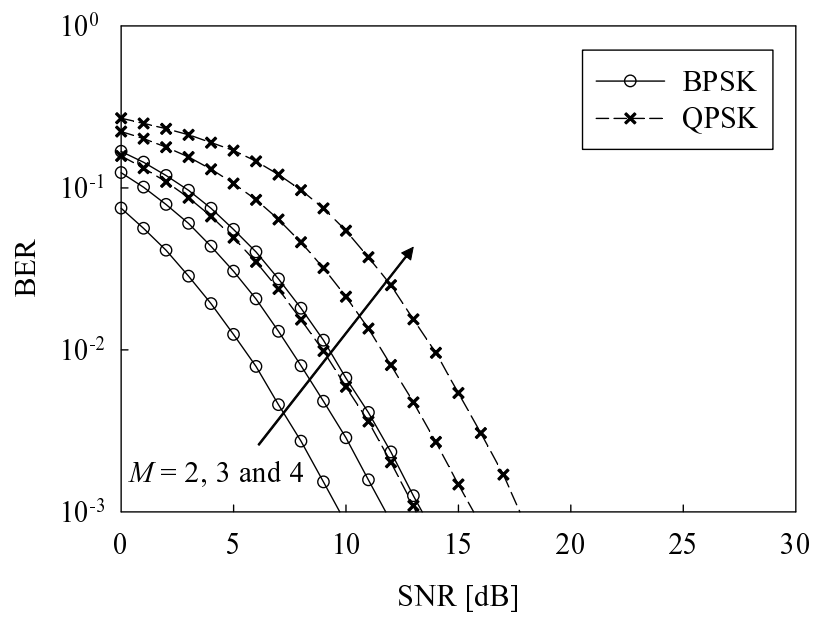

Fig. 8. Achievable BER performance of the uncoded SDM scheme obeying the architecture of Fig. 2 and employing the ML detector in Section III-D, where the number of transmit antennas was varied from $M=2$ to $M=4$, while the number of receive antennas was maintained to be $N=3$. All other system parameters were summarized in Table I.

8 that all the associated BER curves exhibited a good BER performance, without showing any BER floors. Furthermore, it can be seen in Fig. 8 that the ML detector is capable of achieving the maximum attainable diversity order of $M \cdot N$. Upon increasing the multiplexing factor $M$, the performance advantage of the ML detector over the other three linear detectors increased, although this was achieved at the cost of an increased complexity.

Fig. 9 shows the achievable BER performance, comparing the MCMC and the MC-MBER detectors, where the parameters used for the Gibbs-Sampler were given by $N_{\mathrm{MC}}=$ $N_{\mathrm{p}}=2,3,4,5,10,20$ for the MCMC detector as well as by $N_{\mathrm{MC}}=N_{\mathrm{p}}=2,3,4,5$ for the MC-MBER detector. Here, we considered an $(8 \times 8)$-element BPSK-modulated

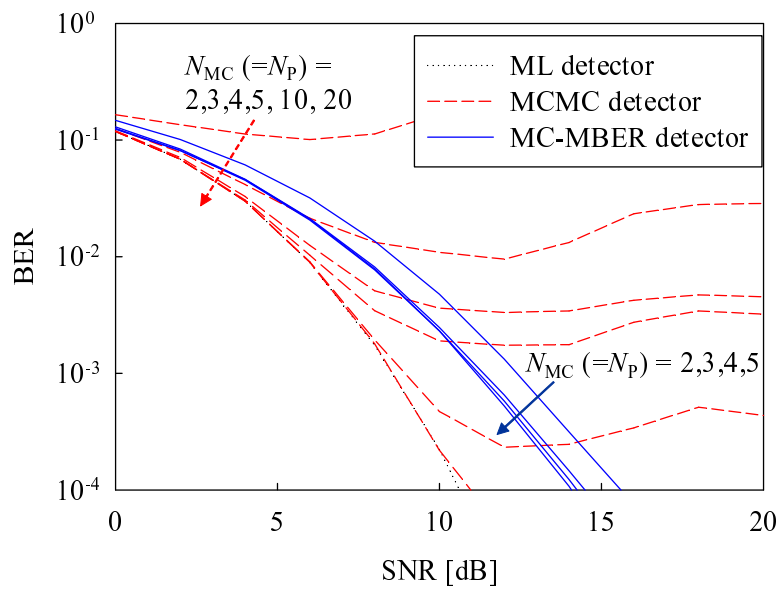

Fig. 9. The achievable BER performance of BPSK-modulated SDM scheme obeying the architecture of Fig. 2, comparing the MCMC and the MCMBER detectors, where the parameters used for Gibbs-Sampler was given by $N_{\mathrm{MC}}=N_{\mathrm{p}}=2,3,4,5,10,20$ for the MCMC detector as well as by $N_{\mathrm{MC}}=N_{\mathrm{p}}=2,3,4,5$ for the MC-MBER detector. Furthermore, the number of both transmit and receive AEs was set to $(M, N)=(8,8)$. All other system parameters were summarized in Table I. The corresponding BER curve of the ML detector was also plotted for comparison.

SDM system, where the number of legitimate sequences was $N_{\mathrm{b}}=2^{8}=256$. The corresponding BER curve of the ML detector was also plotted in Fig. 9 for comparison. Observe in Fig. 9 that upon increasing $N_{\mathrm{MC}}$ for the Gibbs-Sampler to $N_{\mathrm{MC}}=N_{\mathrm{p}}$, the BER of the MCMC detector improved and it converged to that of the ML detector when we have $N_{\mathrm{MC}}=N_{\mathrm{p}}=20$. In other words, it exhibited a performance comparable to that of the ML detector. Nevertheless, when $N_{\mathrm{MC}}\left(=N_{\mathrm{p}}\right)$ was lower than 10 , observe in Fig. 9 that the MCMC detector exhibited an error floor. By contrast, the achievable BER of the MC-MBER detector was good, regardless of the Gibbs-Sampler parameters and it converged at $N_{\mathrm{MC}}=N_{\mathrm{p}}=3$. Since the MC-MBER detector is a hybrid of the statistical and of the deterministic methods, the resultant performance is more robust than that of the purely statistical MCMC detector.

\section{Soft-Input Soft-Output MiMO Detectors}

Having characterized both the achievable performance and the complexity of the class of hard-decision detectors developed for the uncoded SDM system of Fig. 2, we now introduce their soft-information assisted counterparts, in order to create a practical Forward Error Correction (FEC) aided SDM system.

More specifically, we consider the two-stage seriallyconcatenated SDM scheme of Fig. 10, where the uncoded SDM system of Fig. 2 is extended to include a channel encoder and a bit interleaver at the transmitter as well as the corresponding interleaver/deinterleaver and a SISO channel decoder at the receiver, according to the turbo coding principle $[27,50]$. At the transmitter, the source bits are first channel encoded and then interleaved by the interleaver $\Pi$. Then, the interleaved bits are $\mathrm{S} / \mathrm{P}$ converted to $M$ substreams, followed by SDM mapping to each transmit AE. Based on the turbo detection principle, the receiver employs iterative detection in 
This article has been accepted for inclusion in a future issue of this journal. Content is final as presented, with the exception of pagination.
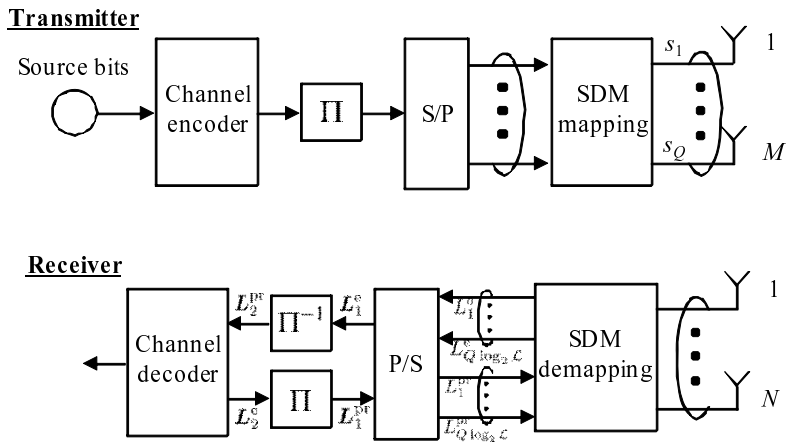

Fig. 10. Schematic of the two-stage serially-concatenated SDM transmitter and receiver, which was developed from its uncoded counterpart of Fig. 2.

the context of the SDM scheme. The receiver consists of two SISO stages, namely the SISO-based detector and the SISO channel decoder. The SISO detector employed demodulates the received symbols $\boldsymbol{Y}$ and outputs the extrinsic information $\boldsymbol{L}_{1}^{\mathrm{e}}$ in the form of LLRs with the aid of the a priori LLRs $\boldsymbol{L}_{1}^{\mathrm{pr}}$, which are fed back from the channel decoders to the detector. The extrinsic LLRs $\boldsymbol{L}_{1}^{\mathrm{e}}$ are then input to the Convolutional Channel (CC) decoders of Fig. 10 after deinterleaving. The extrinsic LLRs $\boldsymbol{L}_{2}^{\mathrm{e}}$ of Fig. 10 are calculated at the channel decoder, output and are interleaved again, before being passed back to the SISO detector component of Fig. 10 as the a priori information. We note that since there is no a priori information during the first iteration, the initial values of $\boldsymbol{L}_{1}^{\mathrm{pr}}$ are set to zero.

Therefore, the SDM demapping block of Fig. 10 has to output bit-wise soft extrinsic information rather than the estimates of the transmitted symbols. To this end, we introduce a range of diverse SISO detectors designed for MIMO systems in this section, including the SISO-MAP detector, the SISOMMSE detector, the SISO-MBER detector, the SISO-MCMC detector and the SISO-MC-MBER detector.

Since the iterative detection is a non-linear process, it is a challenging task to analyze the decoding characteristics and to obtain a closed-form expression for the achievable performance. This also makes it difficult to design the corresponding channel encoder and to determine the appropriate number of iterations at the receiver. Motivated by this problem, ten Brink [51] invented a sophisticated analysis tool, namely the EXIT chart, which visualizes the extrinsic information exchange between the receiver components during the iterative process and enables us to design a near-capacity turbo-coding assisted SDM system. The fundamental theory of EXIT charts will be described later in Section V-A.

Note that while in this treatise we focus our attention on turbo-coded systems as a representative of channel coded systems utilizing soft information, there exist many other soft-information assisted channel-coded systems and their detectors. For example, Low-Density Parity-Check (LDPC) codes [52] constitute another important class of channel codes, where soft information is iteratively exchanged at the decoder, in order to increase the mutual information, similarly to the receiver of turbo-coded systems.

\section{A. SISO MAP Detector}

According to the equivalent system model, the conditional probability $p\left(\boldsymbol{Y} \mid \boldsymbol{S}^{(l)}\right)$ is given by Eq. (1). Considering that the symbol vector $\boldsymbol{S}=\left[s_{1}, s_{2}, \cdots, s_{M}\right]^{\mathrm{T}}$ carries $B$ channelcoded binary bits $\boldsymbol{B}=\left[b_{1}, b_{2}, \cdots, b_{B}\right]^{\mathrm{T}}$, the a posteriori LLR $L_{1}^{\mathrm{po}}$ of the bit $b_{k}(k=1, \cdots, B)$ is given by [53]

$$
\begin{aligned}
L_{1}^{\mathrm{po}}\left(b_{k}\right) & =L_{1}^{\mathrm{po}}\left(b_{k} \mid \boldsymbol{S}\right) \\
& =\underbrace{\ln \frac{\sum_{\boldsymbol{S}^{(l)} \in S_{1}^{k}} p\left(\boldsymbol{Y} \mid \boldsymbol{S}^{(l)}\right) \exp \left(\sum_{j \neq k} b_{j} L_{1}^{\mathrm{pr}}\left(b_{j}\right)\right)}{\sum_{\boldsymbol{S}^{(l)} \in S_{0}^{k}} p\left(\boldsymbol{Y} \mid \boldsymbol{S}^{(l)}\right) \exp \left(\sum_{j \neq k} b_{j} L_{1}^{\mathrm{pr}}\left(b_{j}\right)\right)}}_{L_{1}^{\mathrm{e}}\left(b_{k}\right)} \\
& +L_{1}^{\mathrm{pr}}\left(b_{k}\right),
\end{aligned}
$$

where $S_{i}^{k}(i=0,1)$ indicates a collection of the signals $S$ where the $k$ th bit is $b_{k}=i \in\{0,1\}$. Then, by substituting Eq. (1) into Eq. (6), we arrive at

$$
\begin{aligned}
& L_{1}^{\mathrm{e}}\left(b_{k}\right)= \\
& \ln \frac{\sum_{\boldsymbol{S}^{(l)} \in S_{1}^{k}} \exp \left[-\frac{\left\|\boldsymbol{Y}-\boldsymbol{H} \boldsymbol{S}^{(l)}\right\|^{2}}{N_{0}}+\sum_{j \neq k} b_{j} L_{1}^{\mathrm{pr}}\left(b_{j}\right)\right]}{\sum_{\boldsymbol{S}^{(l)} \in S_{0}^{k}} \exp \left[-\frac{\left\|\boldsymbol{Y}-\boldsymbol{H} \boldsymbol{S}^{(l)}\right\|^{2}}{N_{0}}+\sum_{j \neq k} b_{j} L_{1}^{\mathrm{pr}}\left(b_{j}\right)\right]}(7)
\end{aligned}
$$

Furthermore, using the max-log approximation [54], the extrinsic LLRs $L_{1}^{\mathrm{e}}\left(b_{k}\right)$ may be simplified at the cost of a minimal performance loss.

\section{B. SISO MMSE Detector}

Similarly to the hard-decision MMSE detector reviewed in Section III-B, the SISO MMSE detector is a popular detection scheme, which computes the complex-valued receiver weights by minimizing the expectation of the MSE and then outputs the soft information. At the inner SISO demapping block of Fig. 10, a soft-information vector $\boldsymbol{H} \boldsymbol{S}$, which is calculated based on the a priori information, is subtracted from the equivalent input signals $\boldsymbol{Y}$ excluding the $m$ th multiplexed signal, then the equivalent input signals $\boldsymbol{Y}$ are decontaminated from the effects of the interference by the detector having complexvalued weights as follows: $\hat{s}_{m}=\boldsymbol{w}_{m}^{\mathrm{H}}\left(\boldsymbol{Y}-\boldsymbol{H} \overline{\boldsymbol{S}}+\boldsymbol{h}_{m} \bar{s}_{m}\right)$, where $\overline{\boldsymbol{S}}=\left[\bar{s}_{1}, \bar{s}_{2}, \cdots, \bar{s}_{M}\right]^{\mathrm{T}}$ hosts the signals calculated from the a priori LLRs, whose elements are computed for BPSK as $\bar{s}_{m}=E\left[s_{m}\right]=\tanh \left(\frac{L_{m, 1}^{\mathrm{pr}}}{2}\right)$, and for QPSK as $\bar{s}_{m}=\frac{1}{\sqrt{2}}\left\{\tanh \left(\frac{L_{m, 1}^{\mathrm{pr}}}{2}\right)+j \tanh \left(\frac{L_{m, 2}^{\mathrm{pr}}}{2}\right)\right\}$, where $L_{m, i}^{\mathrm{pr}}(i=$ $\left.1, \cdots, \log _{2} \mathcal{L}\right)$ indicates the bits corresponding to the $m$ th signal $s_{m}$.

Then, by applying to the MMSE criterion mentioned in Section III-B, the weight vector corresponding to the $m$ th substream may be expressed in a closed form as $\boldsymbol{w}_{m}=\left(\boldsymbol{H} \boldsymbol{\Lambda}_{m} \boldsymbol{H}^{\mathrm{H}}+\left|\bar{s}_{m}\right|^{2} \boldsymbol{h}_{m} \boldsymbol{h}_{m}^{\mathrm{H}}+N_{0} \boldsymbol{I}\right)^{-1} \boldsymbol{h}_{m}$, where we have $\boldsymbol{\Lambda}_{m}=\operatorname{diag}\left[v_{1}, \cdots, v_{m-1}, 0, v_{m+1}, \cdots, v_{M}\right]$ and $v_{m}$ represents the variance of the $m$ th substream, calculated from the a priori LLRs as follows: $v_{m}=E\left[\left|s_{m}\right|^{2}\right]-\left|E\left[s_{m}\right]\right|^{2}=1-\left|s_{m}^{-}\right|^{2}$. Assuming that the conditional PDFs $P\left(\hat{s}_{m} \mid b_{m}(i)\right)$ obey the Gaussian distribution, we can express the extrinsic information for BSPK as [28] $L_{1}^{\mathrm{e}}\left(b_{m}(1)\right)=$ $4 \mathcal{R}\left[\boldsymbol{w}_{m}^{\mathrm{H}}\left(\boldsymbol{Y}-\boldsymbol{H} \overline{\boldsymbol{S}}+\boldsymbol{h}_{m} \bar{s}_{m}\right)\right] /\left(1-v_{m} \boldsymbol{w}_{m}^{\mathrm{H}} \boldsymbol{h}_{m}\right)$, 
This article has been accepted for inclusion in a future issue of this journal. Content is final as presented, with the exception of pagination.

and for QPSK as $L_{1}^{\mathrm{e}}\left(b_{m}(1)\right)$

$4 \mathcal{R}\left[\boldsymbol{w}_{m}^{\mathrm{H}}\left(\boldsymbol{Y}-\boldsymbol{H} \overline{\boldsymbol{S}}+\boldsymbol{h}_{m} \bar{s}_{m}\right)\right] /\left(1-v_{m} \boldsymbol{w}_{m}^{\mathrm{H}} \boldsymbol{h}_{m}\right)$

$=$

$L_{1}^{\mathrm{e}}\left(b_{m}(2)\right)=4 \mathcal{I}\left[\boldsymbol{w}_{m}^{\mathrm{H}}\left(\boldsymbol{Y}-\boldsymbol{H} \overline{\boldsymbol{S}}+\boldsymbol{h}_{m} \bar{s}_{m}\right)\right] /\left(1-v_{m} \boldsymbol{w}_{m}^{\mathrm{H}} \boldsymbol{h}_{m}\right)$, where $b_{m}(i)\left(i=1, \cdots, \log _{2} \mathcal{L}\right)$ is the $i$ th channel-coded binary bit of the $m$ th substream at each symbol duration.

\section{SISO MBER Detector}

Similarly to the hard-decision MBER detector introduced in Section III-C, the error probability of the $m$ th substream signal $s_{m}$ can be expressed as a closed form [30] based on Q-function, on $N_{b}=\mathcal{L}^{M}$ number of legitimate transmitted sequences and on signals calculated from the a priori information. Then, the MBER detector's weights are then derived by minimizing the BER function similarly to the harddecision MBER detector. $\boldsymbol{w}_{m}=\arg \min \boldsymbol{w} P_{\mathrm{e}, \mathrm{I}}\left(\boldsymbol{w}_{m}\right)$. The probability $P_{\mathrm{e}, \mathrm{I}}\left(\boldsymbol{w}_{m}\right)$ is a nonlinear function of the weights $\boldsymbol{w}_{m}$, therefore in general the optimization problem has to be solved iteratively. Furthermore, similarly to the SISO MAP detector of Section IV-A, the max-log approximation may be invoked for the sake of reducing the complexity imposed. Moreover, the above-mentioned MBER detector derived for BPSK modulation may be readily extended to multi-level modulation schemes, such as QPSK [55] and QAM [56].

Unfortunately, since the SISO MBER detector has to update the weights per every symbol vector, its total complexity tends to be higher than that of the SISO MAP detector. This may be overcome by introducing MCMC and LLR thresholding techniques as detailed in Section IV-E.

\section{SISO MCMC Detector}

In Section III-E, we have introduced the hard-decision MCMC detector, which applies the hard-decision operation to the output soft information formulated in Eq. (8). The SISO MCMC detector may then be readily implemented by modifying the treatment of the a priori LLRs at the Markov chain sampling operation as well as the max-log approximation [57]:

$$
\begin{aligned}
L_{1}^{\mathrm{e}}\left(b_{k}\right) & =\max _{\boldsymbol{S}^{(i)} \in S_{1}^{k}}\left[-\frac{\left\|\boldsymbol{Y}-\boldsymbol{H} \boldsymbol{S}^{(i)}\right\|^{2}}{N_{0}}+\ln P\left(\boldsymbol{B}_{-k}^{(i)}\right)\right] \\
& -\max _{\boldsymbol{S}^{(i)} \in S_{0}^{k}}\left[-\frac{\left\|\boldsymbol{Y}-\boldsymbol{H} \boldsymbol{S}^{(i)}\right\|^{2}}{N_{0}}+\ln P\left(\boldsymbol{B}_{-k}^{(i)}\right)\right],
\end{aligned}
$$

where $S_{i}^{k}(i=0,1)$ represents a set of samples $\boldsymbol{S}^{(l)}$ generated by the Gibbs-Sampler, whose $k$ th information bit is $b_{k}=i$ and $P\left(\boldsymbol{B}_{-m}^{(i)}\right)$ is the a priori probability, which is calculated from the $a$ priori LLRs.

\section{E. SISO MC-MBER Detector}

As mentioned above in Section IV-C, the high BER performance of the MBER scheme is achieved at the cost of a high computational complexity, which may become particularly challenging in rapidly fading propagation environments, requiring prompt MBER detector weight updates. Similarly, frequent weight-updates are required in iterative detection scenarios, where soft information has to be exchanged between the detector and the channel decoder.
Clearly, the calculation of the MBER weight gradient imposes a high computational complexity, which increases exponentially with the value of $M$. It may be readily shown that an unlikely signal set of $\boldsymbol{S}^{(l)}$ resulting in a small value of $P\left(\boldsymbol{S}^{(l)}\right)$ does not substantially contribute to the gradient expression. Thus, similarly to Section IV-E, we introduce the Markov Chain representation method that efficiently extracts a likely set of signals from the $N_{b}=\mathcal{L}^{M}$ legitimate sequences for the sake of reducing the computational complexity associated with the gradient calculation. This is achieved without degrading the BER performance of the full-complexity SISO MBER scheme. The procedure of the SISO MC-MBER detector is similar to that of the non-iterative hard-decision MC-MBER detector of Fig. 4. The slight difference is in the implementation of the approximate MBER detector block of Fig. 4, where the SISO MBER detector assisted by the a priori LLRs rather than the hard-decision MBER detector is applied based on the $N_{\mathrm{b}}^{\prime}$ most likely signals generated by the GibbsSampler.

Furthermore, the computational complexity of the MCMBER detector is further reduced by introducing a novel concept, namely thetechnique of a priori LLR thresholding, in which a priori information is utilized in the Markov chain simulation in order to reduce the associated computational complexity without substantially degrading its performance.

1) A priori LLR Thresholding Assisted Complexity Reduction: Clearly, the above-mentioned Gibbs-Sampler is largely affected by the a priori information passed to it from the channel decoder, which indicates whether the corresponding bit is more likely to be 1 or 0 . For example, when the LLR $L_{1}^{\mathrm{pr}}\left(b_{k}\right)$ is a large positive value, $P\left(b_{k}^{(i)}=1 \mid \boldsymbol{B}_{-m}^{(i)}, \boldsymbol{Y}, L_{1}^{\mathrm{pr}}\left(b_{k}\right)\right)$ is close to unity. Thus the bit $b_{k}^{(i)}$ is set to 0 as a result of comparing $P\left(b_{k}^{(i)}=1 \mid \boldsymbol{B}_{-k}^{(i)}, \boldsymbol{Y}, L_{1}^{\mathrm{pr}}\left(b_{k}\right)\right)$ to the random variable $\xi$.

Based on this fact, we introduce the novel concept of using an a priori LLR threshold based technique for the GibbsSampler, by omitting the low-probability detection candidates. To be more specific, when the LLR $L_{1}^{\mathrm{pr}}\left(b_{k}\right)$ is higher than a certain threshold value $\zeta>0$, the corresponding bit $b_{k}^{(i)}$ is set to 0 without implementing the Gibbs-Sampling. In the same way, if the LLR $L_{1}^{\mathrm{pr}}\left(b_{k}\right)$ is less than the threshold value $-\zeta$, the bit $b_{k}^{(i)}$ is set to 1 . It is plausible that a lower value of the threshold $\zeta$ leads to a lower complexity at the cost of a less accurate approximation of the Gibbs-Sampler.

To elaborate a little further, the complexity reduction effects of the a priori LLR thresholding technique is substantially affected by the a priori LLRs utilized. For example, since no a priori LLRs can be given in the first iteration, we cannot benefit from this technique at this stage. After the first iteration, upon increasing the number of the iterations, the absolute value of the a priori LLRs gleaned from the channel decoder tend to increase, leading to the increase in the effects of the computational complexity reduction advocated by the $a$ priori LLR thresholding technique. Therefore, the beneficial effect of this technique on the computational complexity reduction largely depends on the number of iterations as well as on the SNR. 
This article has been accepted for inclusion in a future issue of this journal. Content is final as presented, with the exception of pagination.

a priori LLR $\boldsymbol{L}^{\mathbf{p r}}$

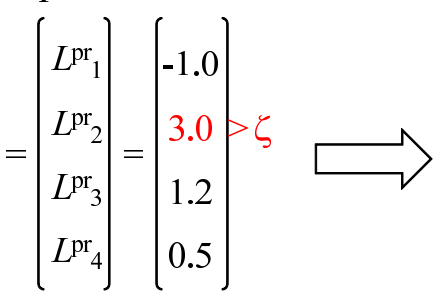

$N_{\mathrm{MC}}$ number of Generated signals

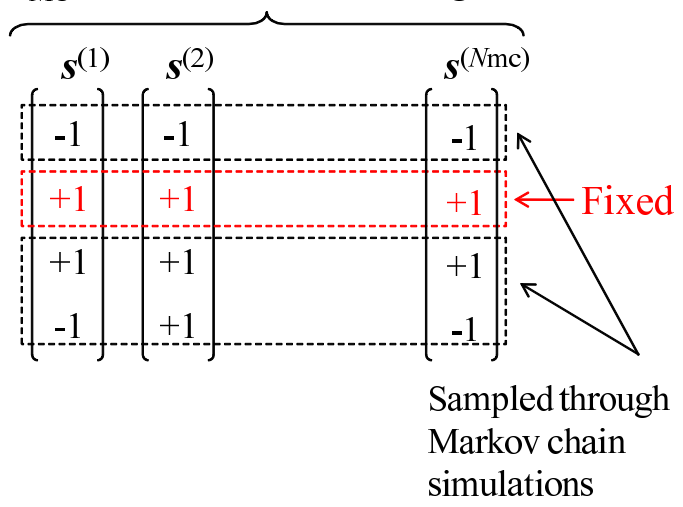

Fig. 11. Explanation of the a priori LLR thresholding technique, where the threshold value $\zeta$ was set to $\zeta=3$.

\section{F. Computational Complexity}

In this section, we compare the computational complexity of the SISO detectors, such as the SISO MBER detector, the SISO MAP detector, the SISO MCMC detector and the SISO MC-MBER detector, which have to calculate $M \log _{2} \mathcal{L}$ LLRs corresponding to the information bits transmitted during each symbol interval. Since the basic calculations associated with each SISO detectors' complexity is similar to those of the hard-decision detectors shown in Section III, we only list the results in Table VIII. The corresponding computational complexity is evaluated in terms of the real-valued multiplications. Here, we show both the complexity of the MCMBER detectors, either employing or dispensing with the $a$ priori LLR thresholding technique of Section IV-E1, where $\gamma$ represents the relative frequency of activating the LLR thresholding during the implementation of the Gibbs-Sampler.

We note that except for the SISO MMSE detector, the employment of the max-log approximation was assumed for the LLR calculations, which substantially reduces the associated complexity in comparison to the exact calculations of the LLR values.

\section{EXIT Chart Aided System Design}

In this section, we firstly highlight the concept of EXIT charts, which is a powerful technique used for analyzing the convergence behaviour of iterative detection aided transmissions based on the turbo-coding principle. EXIT chart in the next section. The EXIT charts are then used to analyze the SDM system detected with the aid of SISO detectors introduced in the previous section.

\section{A. EXIT Chart}

In turbo detection, an infinitesimally low BER may be attained by the iterative exchange of extrinsic mutual information between two SISO decoders, i.e. the inner and outer decoders. Since the iterative decoding process is not linear, the prediction of its convergence behaviour is a challenging task. The ingenious tool of EXIT charts was proposed by ten Brink $[51,58]$ for the visualization of the iterative decoding behaviour and for the prediction of the 'BER-cliff' position, where the BER suddenly drops. More specifically, the input/output relationship of the mutual information at each decoder is characterized by the EXIT chart and then their interaction assisted by the iterative decoding process is examined without time-consuming bit-by-bit Monte-Carlo simulations.

The SDM demapper of Fig. 10 outputs the a posteriori information based on the noise-contaminated channel output observations and on the a priori information gleaned from the outer channel decoder. By subtracting the a priori information from the a posteriori information, the SDM demapper generates the extrinsic information. The EXIT chart analysis relies on exploiting the following two assumptions. Firstly, it assumes having a sufficiently long interleaver that assures statistical independence of the a priori LLRs $L^{\mathrm{a}}$. Secondly, it stipulates having a Gaussian distribution for the LLRs, which may be formulated as $L^{\mathrm{a}}=\mu_{A} \cdot s+n_{A}$, where we have $\mu_{A}=\sigma_{A}^{2} / 2$, whith $\mu$ being the variance of the LLRs $L^{\mathrm{a}}$. Here the conditional probability of the a priori LLR $L^{\mathrm{a}}$ is given by

$$
p_{L^{\mathrm{a}}}(x \mid S=s)=\frac{1}{\sqrt{2 \pi} \sigma_{A}} \exp \left(-\frac{\left(x-\left(\sigma_{A}^{2} / 2\right) \cdot s\right)^{2}}{2 \sigma_{A}^{2}}\right) .
$$

In binary EXIT chart analysis, the mutual information between the a priori or extrinsic LLRs $L^{i}(i=\mathrm{a}, \mathrm{e})$ and the corresponding bits $S$ is calculated by the following equation [51]

$$
I\left(L^{i} ; S\right)=\frac{1}{2} \sum_{s= \pm 1} \int_{-\infty}^{\infty} p_{L^{i}}(x \mid s) \log \frac{p_{L^{i}}(x \mid s)}{p_{L^{i}}(x)} d x
$$

with $p_{L^{i}}(x)=\frac{1}{2}\left\{p_{L^{i}}(x \mid s=+1)+p_{L^{i}}(x \mid s=-1)\right\}$, where $p_{L^{i}}(x \mid s)$ is the probability of the a priori information conditioned on encountering $s= \pm 1$. Then, by applying Eq. (9), which is based on the Gaussian distribution assumption to the PDF of the LLRs $L^{\mathrm{a}}$, the mutual information between the $a$ priori LLRs $L^{\mathrm{a}}$ and the equiprovable transmitted symbols $s$ can be written by [51]

$$
\begin{aligned}
I\left(L^{\mathrm{a}} ; S\right) & =I_{A}\left(\sigma_{A}\right) \\
& =1-\int_{-\infty}^{\infty} \frac{1}{\sqrt{2 \pi \sigma_{A}^{2}}} \exp \left(-\frac{\left(L^{\mathrm{a}}-\sigma_{A}^{2} / 2\right)^{2}}{2 \sigma_{A}^{2}}\right) \\
& \times \log _{2}\left(1+e^{-L}\right) d L .
\end{aligned}
$$

For simplicity, Eq. (11) is written as $I=J\left(\sigma_{A}\right)$, which 
This article has been accepted for inclusion in a future issue of this journal. Content is final as presented, with the exception of pagination.

TABLE VIII

COMPUTATIONAL COMPLEXITY OF THE SISO MMSE, THE SISO MBER, THE SISO MAP, THE SISO MCMC AND THE SISO MC-MBER DETECTORS FOR THE TWO-STAGE TURBO-CODED SDM SYSTEM OF FIG. 10

\begin{tabular}{|c|c|c|}
\hline MUD & Computational complexity & \\
\hline 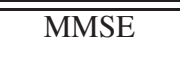 & $\begin{aligned}\left(4 M N^{2}+2 M N+10 N^{2}-2 N+\right. & \left.2 \log _{2} \mathcal{L}+1\right) M \\
& +12 M N+5 M\end{aligned}$ & $\begin{array}{r}\text { (weight calculation) } \\
\text { (LLR calculation) }\end{array}$ \\
\hline MBER & $\begin{array}{r}\log _{2} \mathcal{L} \cdot N_{\mathrm{g}} N_{\mathrm{b}} M\left(8 M N+10 N+M \log _{2} \mathcal{L}+5\right) \\
+3 M \log _{2} \mathcal{L}+N_{\mathrm{g}} M(8 M+2) \\
+M \log _{2} \mathcal{L} N_{\mathrm{b}}(4 N+2)\end{array}$ & $\begin{array}{r}\text { (weight calculation) } \\
\text { (LLR calculation) }\end{array}$ \\
\hline MAP & $N_{\mathrm{b}}(4 M N+2 N+1)+M \log _{2} \mathcal{L}$ & \\
\hline MCMC & $\begin{aligned} 4\left(1+\log _{2} \mathcal{L} \cdot\right. & \left.M N_{\mathrm{MC}} N_{\mathrm{P}}\right) M(N+1) \\
& +2 \log _{2} \mathcal{L} \cdot M N_{\mathrm{MC}} N_{\mathrm{P}} \\
& +2 N_{\mathrm{b}}^{\prime}\left(M \log _{2} \mathcal{L}-1\right)\end{aligned}$ & $\begin{array}{l}\text { (Gibbs-Sampler) } \\
\text { (LLR calculation) }\end{array}$ \\
\hline $\begin{array}{l}\text { MC-MBER } \\
\text { (without } \\
\text { thresholding) }\end{array}$ & $\begin{aligned} & 4\left(1+\log _{2} \mathcal{L} \cdot\right.\left.M N_{\mathrm{MC}} N_{\mathrm{P}}\right) M(N+1) \\
&+2 \log _{2} \mathcal{L} \cdot M N_{\mathrm{MC}} N_{\mathrm{P}} \\
&+\log _{2} \mathcal{L} \cdot N_{\mathrm{g}} N_{\mathrm{b}}^{\prime} M\left(8 M N+10 N+M \log _{2} \mathcal{L}+5\right) \\
&+3 M \log _{2} \mathcal{L}+N_{\mathrm{g}} M(8 M+2) \\
&+M \log _{2} \mathcal{L} N_{\mathrm{b}}^{\prime}(4 N+2)\end{aligned}$ & $\begin{array}{l}\text { (Gibbs-Sampler) } \\
\text { (approx. MBER) } \\
\text { (LLR calculation) }\end{array}$ \\
\hline $\begin{array}{l}\text { MC-MBER } \\
\text { (with } \\
\text { thresholding) }\end{array}$ & $\begin{aligned} 4\left[1+\log _{2} \mathcal{L} \cdot\right. & \left.M(1-\gamma) N_{\mathrm{MC}} N_{\mathrm{P}}\right] M(N+1) \\
& +2 \log _{2} \mathcal{L} \cdot M(1-\gamma) N_{\mathrm{MC}} N_{\mathrm{P}} \\
+\log _{2} \mathcal{L} \cdot N_{\mathrm{g}} N_{\mathrm{b}}^{\prime} M( & \left.8 M N+10 N+M \log _{2} \mathcal{L}+5\right) \\
+ & +3 M \log _{2} \mathcal{L}+N_{\mathrm{g}} M(8 M+2) \\
& +M \log _{2} \mathcal{L} N_{\mathrm{b}}^{\prime}(4 N+2)\end{aligned}$ & $\begin{array}{l}\text { (Gibbs-Sampler) } \\
\text { (approx. MBER) } \\
\text { (LLR calculation) }\end{array}$ \\
\hline
\end{tabular}

we refer to as the $J$-function. In [59], this $J$-function was approximated by

$$
I=J\left(\sigma_{A}\right) \approx\left(1-2^{-H_{1} \sigma_{A}^{2 H_{2}}}\right)^{H_{3}},
$$

where the constants $H_{1}, H_{2}$ and $H_{3}$ are given by 0.3073 , 0.9835 and 1.1064 , respectively. The $J$-function is a unique unambiguous function, and thus its inverse function is determined uniquely as

$$
\sigma_{A}=J^{-1}(I) \approx\left(-\frac{1}{H_{1}} \log \left(1-I^{1 / H_{3}}\right)\right)^{\frac{1}{2 H_{2}}}
$$

By contrast, the mutual information between the extrinsic LLRs $L^{\mathrm{e}}$ and the corresponding bits $S$ is calculated by the experimentally gathered histogram. More specifically, the LLRs $L^{\mathrm{a}}$ generated by using the covariance $\sigma_{A}$ in (13) are input to the corresponding SISO decoder, and then the histogram of the output LLRs $L^{\mathrm{e}}$ is evaluated in order to obtain the $\operatorname{PDF} p_{L^{\mathrm{e}}}(x \mid s)$. Finally, the mutual information $I_{E}$ is calculated using Eq. (10). According to this calculation procedure, the two EXIT curves, i.e. the inner and outer decoder's EXIT curves, may be drawn. By examining the relationship of the two curves, we can predict the convergence characteristics of the iterative receiver.

The inner and outer decoders' EXIT curves should not intersect before the point of $\left(I_{\mathrm{A}}, I_{\mathrm{E}}\right)=(1.0,1.0)$, which leads to the ideal extrinsic information exchange between the two decoders. The emergence of an open EXIT chart convergence tunnel enables the system to achieve an infinitesimally low BER at the corresponding SNR. However, since in general the outer decoder's EXIT curve is not guaranteed to reach the point of perfect convergence to a vanishingly low BER at $\left(I_{\mathrm{A}}, I_{\mathrm{E}}\right)=(1.0,1.0)$ due to the non-recursive nature of the inner code, this ideal condition may not always be satisfied, depending on the system's structure. Nevertheless, every system has the potential of arriving at a point of $\left(I_{\mathrm{A}}, I_{\mathrm{E}}\right)=$ $(1.0, \alpha)$ associated with $0<\alpha<1$, if the two EXIT curves

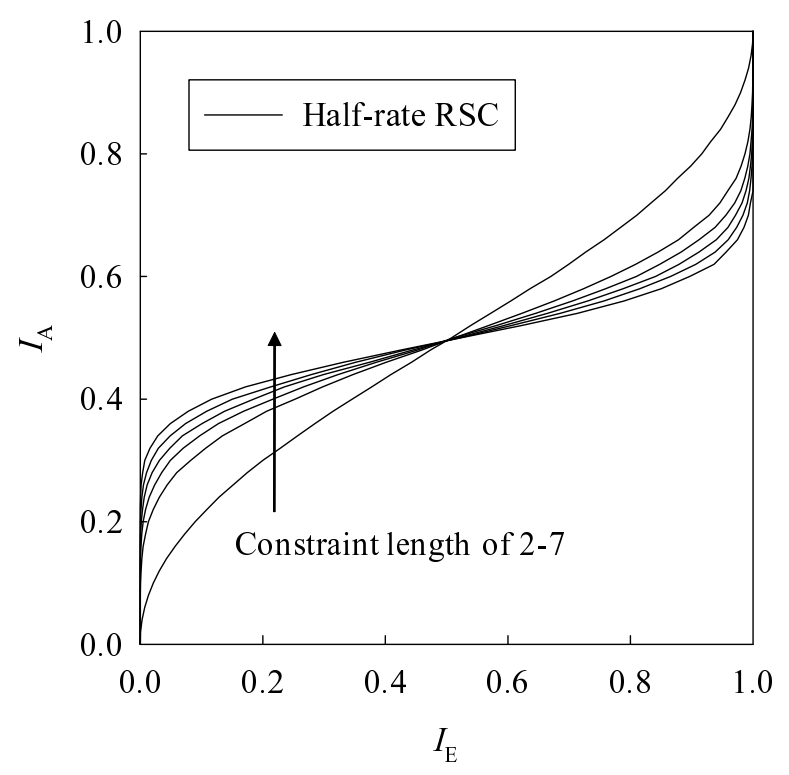

Fig. 12. Outer decoder EXIT curves corresponding to a half-rate RSC having the constraint length of 2 to 7, obeying the generator polynomials shown in Table IX.

form an open tunnel, where we can have a moderately low BER.

Fig. 12 shows the six different outer decoder EXIT curves corresponding to various half-rate RSC codes having the constraint lengths spanning from 2 to 7 , obeying the generator polynomials shown in Table IX. The generator polynomials $\left(G_{\mathrm{r}}, G\right)_{8}$ employed in the simulation are listed in Table IX, which are represented in octal form.

It can be seen from Fig. 12 that upon increasing the constraint length of the RSC, the slope of the EXIT curve becomes less steep around the value of $I_{\mathrm{A}}=0.5$. Thus, for $I_{\mathrm{A}}>0.5$, a RSC code having a higher constraint length has 
This article has been accepted for inclusion in a future issue of this journal. Content is final as presented, with the exception of pagination.

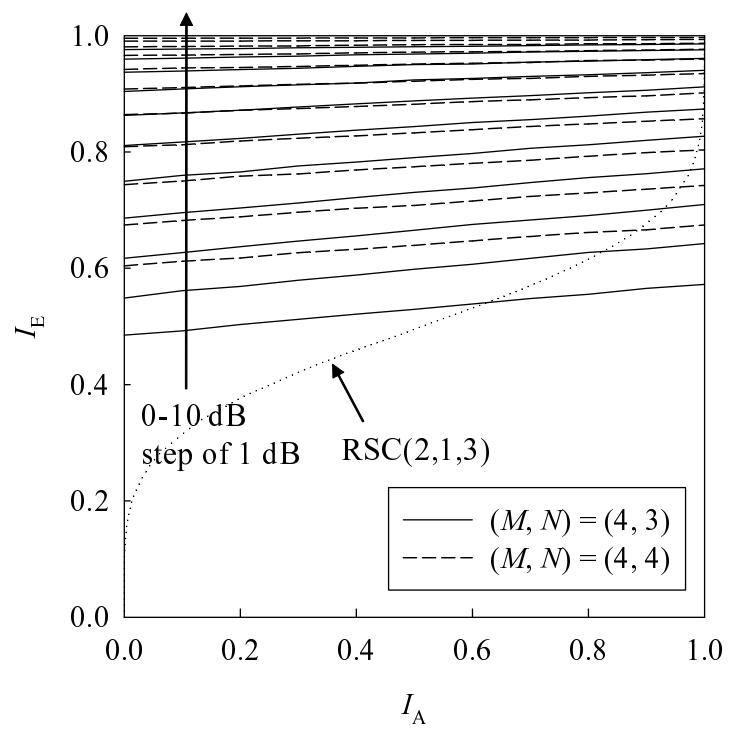

(a) BPSK

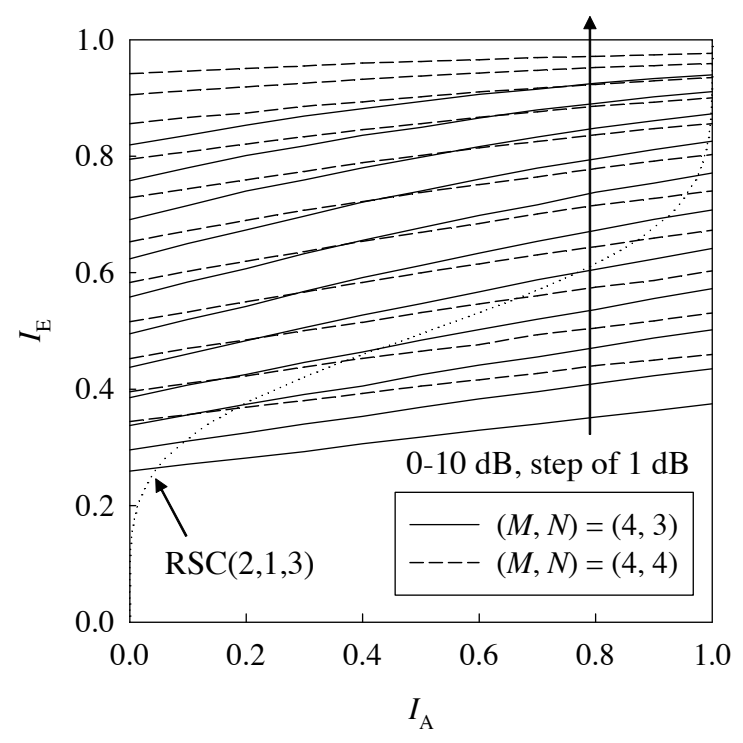

(b) QPSK

Fig. 13. The inner code's EXIT curves of the SISO MAP detector in the serially-concatenated SDM system seen in Fig. 10, for the scenarios of $(M, N)=$ $(4,3)$ AEs and of $(M, N)=(4,4)$ AEs. Here, the SNR was varied from $0 \mathrm{~dB}$ to $10 \mathrm{~dB}$ in every $1 \mathrm{~dB}$. We also plotted the outer EXIT curve of the half-rate $\operatorname{RSC}(2,1,3)$ with generator polynomials of $\left(G_{\mathrm{r}}, G\right)=(5,7)_{8}$ in octal form. All other system parameters were summarized in Table X.

TABLE IX

GENERATOR POLYNOMials EMPLOYED FOR THE HALF-RATE RSC CODES

\begin{tabular}{|c|c|}
\hline $\begin{array}{c}\text { Constraint } \\
\text { length }\end{array}$ & $\begin{array}{c}\text { Generator polynomials } \\
\text { in octal }\left(G_{\mathrm{r}}, G\right)_{8}\end{array}$ \\
\hline \hline 2 & $(3,2)_{8}$ \\
3 & $(5,7)_{8}$ \\
4 & $(15,17)_{8}$ \\
5 & $(35,23)_{8}$ \\
6 & $(53,75)_{8}$ \\
7 & $(133,171)_{8}$ \\
8 & $(247,371)_{8}$ \\
9 & $(561,753)_{8}$ \\
\hline
\end{tabular}

the potential of exhibiting a wider EXIT tunnel. This indicates that depending on the parameters of the RSC code employed, the characteristics of the iterative receiver change and hence the choice of these parameters provide us with an additional degree of design freedom.

In contrast to the outer code's EXIT chart of Fig. 12, Figs 13(a) and 13(b) show the inner code's EXIT curves of the BPSK- and QPSK-modulated SDM system seen in Fig. 10, for the scenarios of $(M, N)=(4,3)$ AEs and $(M, N)=(4,4)$ AEs. Here, we employed the SISO MAP detector of Section IV-A and the SNR value was varied from $0 \mathrm{~dB}$ to $10 \mathrm{~dB}$ with the step size of $1 \mathrm{~dB}$. The outer RSC $(2,1,3)$ decoder's EXIT curve was also plotted. Observe in Figs 13(a) and 13(b) that upon increasing the SNR value, the inner decoder's EXIT curve moved upwards, hence leading to a wider EXIT tunnel. Additionally, it was found that as expected, a increase in the constellation size $\mathcal{L}$ and an decrease in the number of receive antennas $N$ degrades the corresponding inner code's EXIT curve by shifting it downwards.

Having generated the EXIT charts for the SDM system invoking the SISO-MAP detector, we then embark on veri-
TABLE $X$

BASIC SYSTEM PARAMETERS OF THE CODED SDM SCHEME OF FIG. 10.

\begin{tabular}{lr}
\hline \hline Number of transmit antennas & $M=4$ \\
Number of receive antennas & $N=3,4$ \\
Modulation & BPSK, QPSK \\
Channels & Frequency-flat Rayleigh fading \\
Channel's coherence-time & $\tau=1$ symbol duration \\
& \\
Interleaver blocklength & 200000 bits \\
Channel code & RSC $(2,1,3)$ \\
Generator polynomials & $\left(G_{\mathrm{r}}, G\right)=(5,7)_{8}$ \\
Number of iterations & $I=10$ \\
\hline \hline
\end{tabular}

fying the prediction of the iterative convergence by drawing the decoding trajectory within the EXIT chart. Fig. 14 shows the decoding trajectory corresponding to the interleaver length of 200000 bits at SNR $=4 \mathrm{~dB}$. Ovserve in Fig. 14 that the trajectory exhibited a good match with the prediction, where the iterative decoding converged after $I=5$ iterations. Furthermore, the achievable BER performance is shown in Fig. 15, where the number of iterations $I$ was changed from $I=0$ to $I=10$.

In order to provide further insights, let us now characterize the convergence behaviour of other SISO detectors. Firstly, Fig. 16 show the EXIT charts for the SISO MMSE detector of Section IV-B and for the SISO MBER detector of Section IV-C. Here, we considered the scenario of $(M, N)=(4,3)$ AEs and BPSK modulation. It can be seen that the EXIT curve of the SISO MBER detector exhibited a wider EXIT tunnel than that of the SISO MMSE detector especially for BPSK, although both the MBER and MMSE schemes' EXIT 
This article has been accepted for inclusion in a future issue of this journal. Content is final as presented, with the exception of pagination.

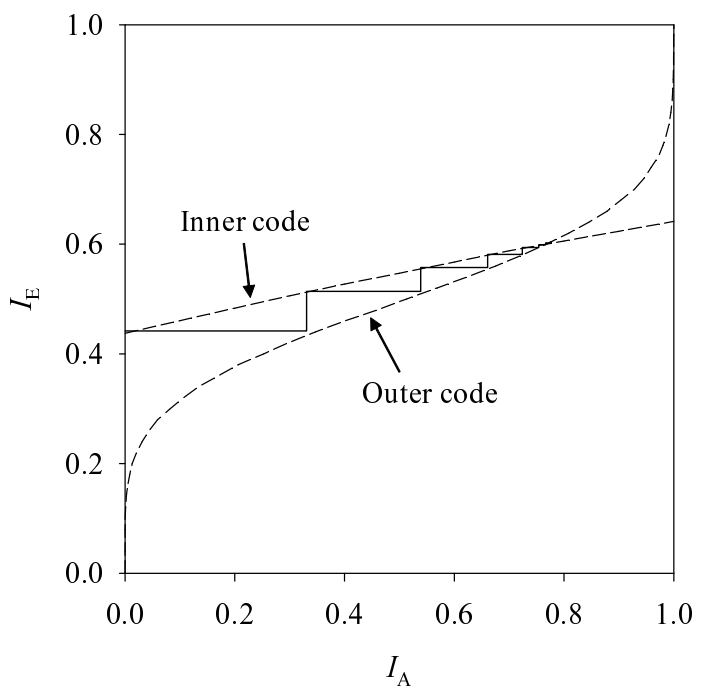

Fig. 14. Decoding trajectory of the iteratively detected half-rate RSC-coded QPSK-modulated SDM system of Fig. 10 employing $(M, N)=(4,3)$ AEs and the SISO MAP detector of Section IV-A. The interleaver lengths was set to 200000 bits, while the SNR was $4 \mathrm{~dB}$.

curves predicted a lower BER performance than that of the SISO MAP detector. ${ }^{3}$

Fig. 17 shows the MCMC detector's EXIT curves for different values of $N_{\mathrm{MC}}\left(=N_{\mathrm{P}}\right)$, which was varied from $N_{\mathrm{MC}}=N_{\mathrm{P}}=2$ to $N_{\mathrm{MC}}=N_{\mathrm{P}}=10$, at $\mathrm{SNR}=5 \mathrm{~dB}$. Observe in Fig. 17 that upon increasing the value of $N_{\mathrm{MC}}\left(=N_{\mathrm{P}}\right)$, the EXIT curve improved, reaching that of the MAP detector for $N_{\mathrm{MC}}=N_{\mathrm{P}}=10$. Furthermore, Fig. 18 shows the computational complexity imposed by the MCMC detector, which corresponds to the EXIT curve of Fig. 17. Although upon increasing the $N_{\mathrm{MC}}\left(=N_{\mathrm{P}}\right)$ the computational complexity also increased, the complexity of the MCMC detector remained ten times lower than that of the MAP detector for $N_{\mathrm{MC}}=N_{\mathrm{P}}=10$. According to Figs. 17 and 18, this suggests that the MCMC detector constitutes a good approximation of the MAP detector, provided that a sufficiently high number of samples are generated by the Gibbs-Sampler.

Fig. 19 shows the EXIT curves of the MC-MBER detector at $\mathrm{SNR}=5 \mathrm{~dB}$, while deactivating the a priori LLR thresholding technique of Section IV-E1, where the GibbsSampler parameters were chosen as $N_{\mathrm{MC}}=N_{\mathrm{P}}=2,3,4$ and 5. Furthermore, we plotted the corresponding EXIT curve of the full-complexity MBER detector in order to characterize the upper bound of the MC-MBER detector. Recall that the MC-MBER detector is the reduced-complexity version of the full-complexity MBER detector. Observe in Fig. 19 that upon increasing the values $N_{\mathrm{MC}}\left(=N_{\mathrm{P}}\right)$, the corresponding

\footnotetext{
${ }^{3}$ In order to provide further insights, consider the special case of the twostage receiver of Fig. 10, where the number of iterations $I$ is set to zero, which constitutes the two-stage non-iterative receiver. Since in this scenario the SDM demapping block of Fig. 10 does not utilize a priori information, the linear weights remains constant for the linear SISO detectors, such as the SISO MMSE and the SISO MBER detectors. Therefore, the associated complexity may be substantially reduced at the cost of no iterative gain, where the extrinsic information output from the SDM demapping block corresponds to the point of $I_{\mathrm{A}}=0$.
}

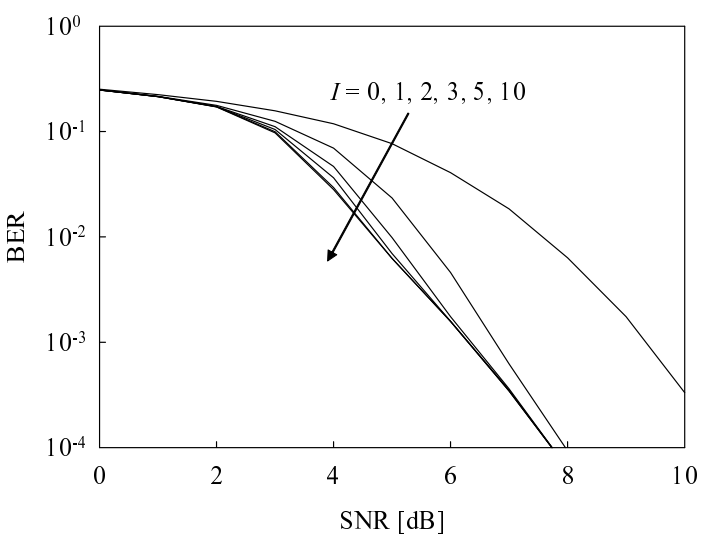

Fig. 15. Achievable BER performance of the iteratively detected half-rate RSC-coded QPSK modulated SDM system of Fig. 10 employing $(M, N)=$ $(4,3)$ AEs and the SISO MAP detector of Section IV-A, where the number of iterations $I$ was varied from $I=0$ to $I=10$, while the interleaver length was set to 200000 bits. All other system parameters were summarized in Table X.

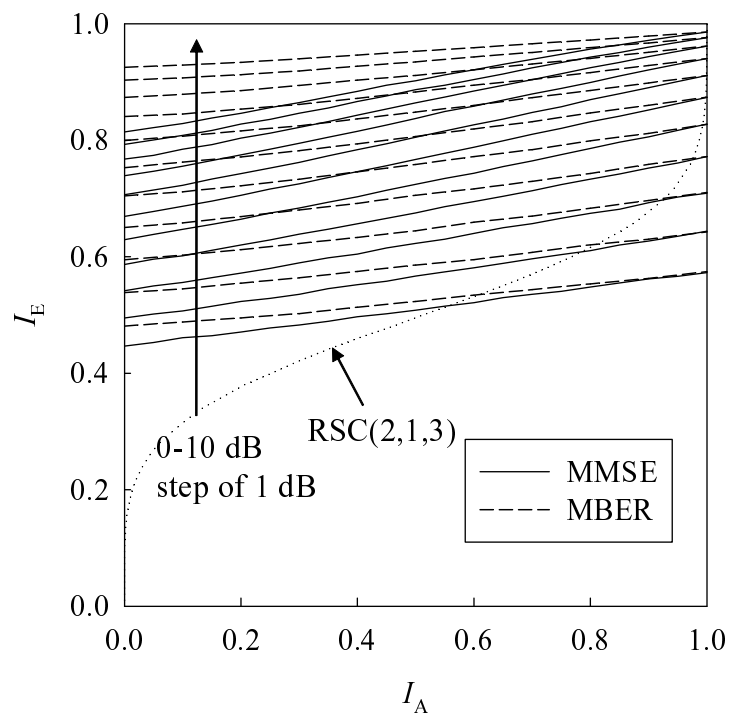

Fig. 16. The inner code's EXIT curves of the SISO MMSE and MBER detectors in the serially-concatenated BPSK-modulated SDM system seen in Fig. 10, for the scenario of $(M, N)=(4,3)$ AEs. Here, the SNR was varied from $0 \mathrm{~dB}$ to $10 \mathrm{~dB}$ in every $1 \mathrm{~dB}$. We also plotted the outer EXIT curve of the half-rate $\operatorname{RSC}(2,1,3)$ with generator polynomials of $\left(G_{\mathrm{r}}, G\right)=(5,7)_{8}$ in octal form. All other system parameters were summarized in Table X.

EXIT curve of the MC-MBER detector was shifted slightly higher, mainly in the low $I_{\mathrm{A}}$ regime. More specifically, the EXIT curve recorded for the case of $N_{\mathrm{MC}}=N_{\mathrm{P}}=5$ attained a slightly lower performance than that of the fullcomplexity MBER detector. Accordingly, it can be argued that the proposed MC-MBER detector was capable of efficiently approximating the full-complexity MBER detector. We also note that while the performance of the MCMC detector was substantially affected by the Gibbs-Sampler's parameters, as evidenced by Fig. 17, the MC-MBER detector exhibited a good performance for low $N_{\mathrm{MC}}=N_{\mathrm{P}}$ values, as shown in Fig. 19. This is because the MC-MBER detector is a 
This article has been accepted for inclusion in a future issue of this journal. Content is final as presented, with the exception of pagination.

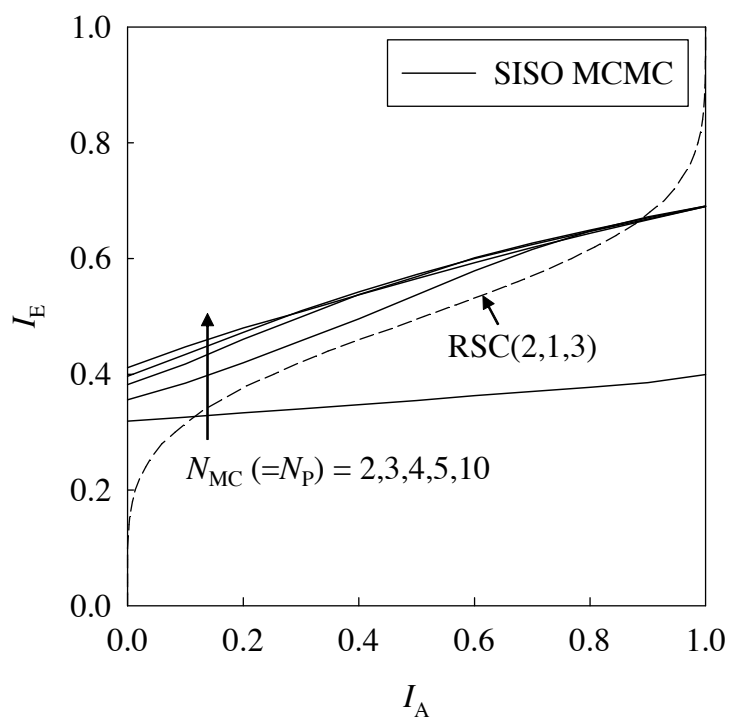

Fig. 17. EXIT chart of the SISO MCMC detector in the QPSK-modulated $(6 \times 4)$-element SDM system of Fig. 10 at the $\mathrm{SNR}=5 \mathrm{~dB}$, comparing the effects of the Gibbs-Sampling parameters, where $N_{\mathrm{MC}}\left(=N_{\mathrm{P}}\right)$ was given by $N_{\mathrm{MC}}=N_{\mathrm{P}}=2,3,4,5,10$. All other system parameters were summarized in Table X.

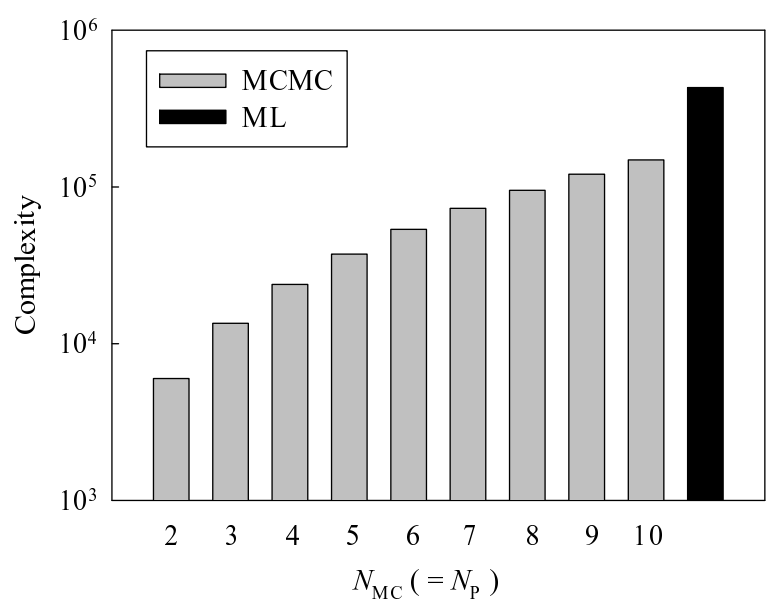

Fig. 18. Computational complexity imposed by the SISO-MCMC detector in the QPSK-modulated $(6 \times 4)$-element SDM system of Fig. 10 at the SNR $=5$ $\mathrm{dB}$, comparing the effects of the Gibbs-Sampling parameters, where $N_{\mathrm{MC}}(=$ $N_{\mathrm{P}}$ ) was given by $N_{\mathrm{MC}}=N_{\mathrm{P}}=2,3,4,5,10$. All other system parameters were summarized in Table $\mathrm{X}$.

stochastic-deterministic hybrid detector, rather than being a purely stochastic detector. Next, in Fig. 20 we characterized the computational complexity imposed by the SISO MCMBER detector, which corresponds to the EXIT curves of Fig. 19. Here, the number of iterations $I$ was varied from $I=0$ to $I=10$, noting that the number of sampled signals $N_{\mathrm{b}}^{\prime}$ required is also affected by the a priori information gleaned from the channel decoder. It can be seen from Fig. 20 that the complexity of the MC-MBER detector employing the GibbsSampler parameter of $N_{\mathrm{MC}}=N_{\mathrm{P}} \leq 5$ exhibited more than ten times lower complexity than that of the MAP detector. Additionally, upon increasing the number of iterations $I$, the

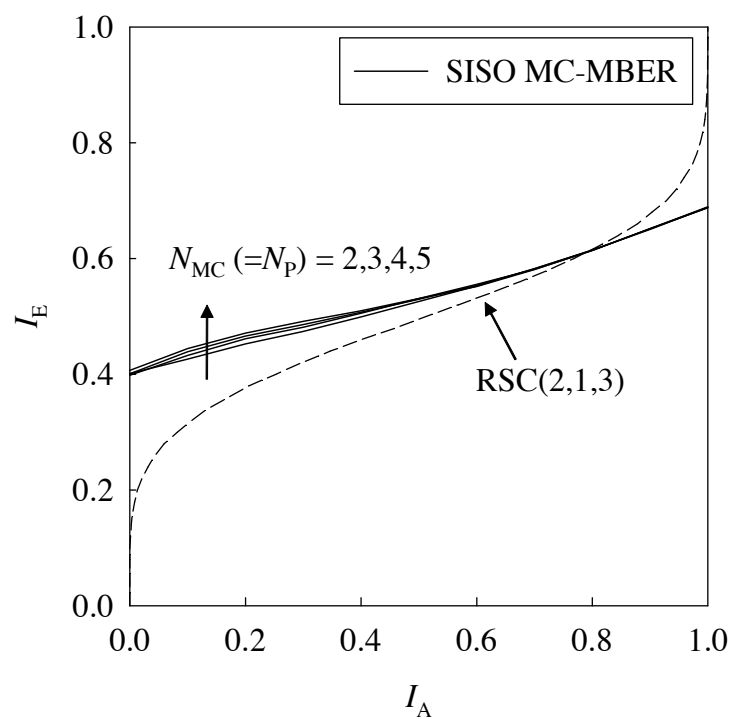

Fig. 19. EXIT chart of the MC-MBER detector in the QPSK-modulated $(6 \times 4)$-element SDM system of Fig. 10 at the SNR $=5 \mathrm{~dB}$, comparing the effects of the Gibbs-Sampling parameters, where $N_{\mathrm{MC}}\left(=N_{\mathrm{P}}\right)$ was given by $N_{\mathrm{MC}}=N_{\mathrm{P}}=2,3,4,5$, while the $a$ priori LLR thresholding was deactivated. All other system parameters were summarized in Table X.

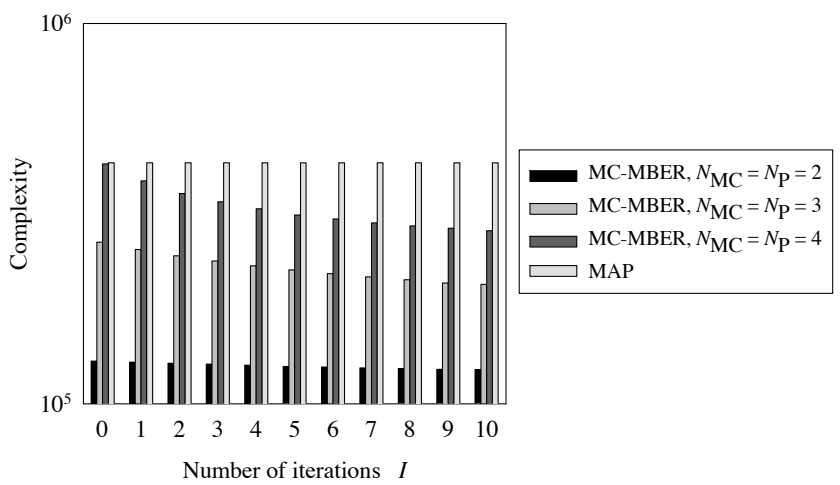

Fig. 20. Computational complexity imposed by the SISO MC-MBER detector in the QPSK-modulated $(6 \times 4)$-element SDM system of Fig. 10 at the $\mathrm{SNR}=5 \mathrm{~dB}$, comparing the effects of the Gibbs-Sampling parameters, where $N_{\mathrm{MC}}\left(=N_{\mathrm{P}}\right)$ was given by $N_{\mathrm{MC}}=N_{\mathrm{P}}=2,3,4,5$, while the $a$ priori LLR thresholding was deactivated. All other system parameters were summarized in Table X.

complexity of the MC-MBER detector was reduced owing to the beneficial effects of having higher a priori LLRs. ${ }^{45}$

Furthermore, in Fig. 21 we investigated the a priori LLR thresholding technique proposed for the complexity reduction of the MC-MBER detector, where the EXIT curves of the MCMBER detector employing the different threshold values of $\zeta=0.5,1,5$ and 10 were considered, for the QPSK-modulated $(6 \times 4)$-element $\mathrm{SDM}$ system at $\mathrm{SNR}=5 \mathrm{~dB}$. It was found

${ }^{4}$ To elaborate a little further, the a priori LLR thresholding technique was found to further reduce the complexity of the MC-MBER detector, although the detailed simulation results are omitted for the sake of saving space.

${ }^{5}$ Although in this paper we considered low-order constellations for the sake of simplicity, the concepts of the MCMC and the MC-MBER detectors may be readily applicable to higher order constellation schemes, such as 16-QAM and 64-QAM. Further details and numerical results can be found in [32] for the MCMC detector and in [60] for the MC-MBER detector. 
This article has been accepted for inclusion in a future issue of this journal. Content is final as presented, with the exception of pagination.

TABLE XI

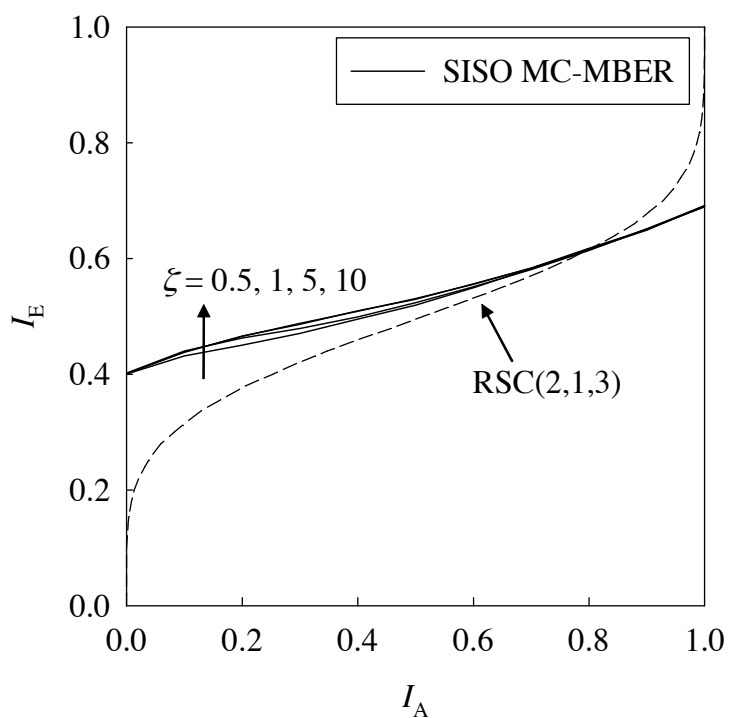

Fig. 21. EXIT curves of the MC-MBER detector in the QPSK-modulated $(6 \times 4)$-element $\mathrm{SDM}$ system of Fig. 10 at the $\mathrm{SNR}=5 \mathrm{~dB}$, where the $a$ priori LLR thresholding was activated with the aid of the threshold values of $\zeta=0.1,1,5$ and 10 . Here, the Gibbs-Sampling parameters was set to $N_{\mathrm{MC}}=N_{\mathrm{P}}=10$. All other system parameters were summarized in Table $\mathrm{X}$.

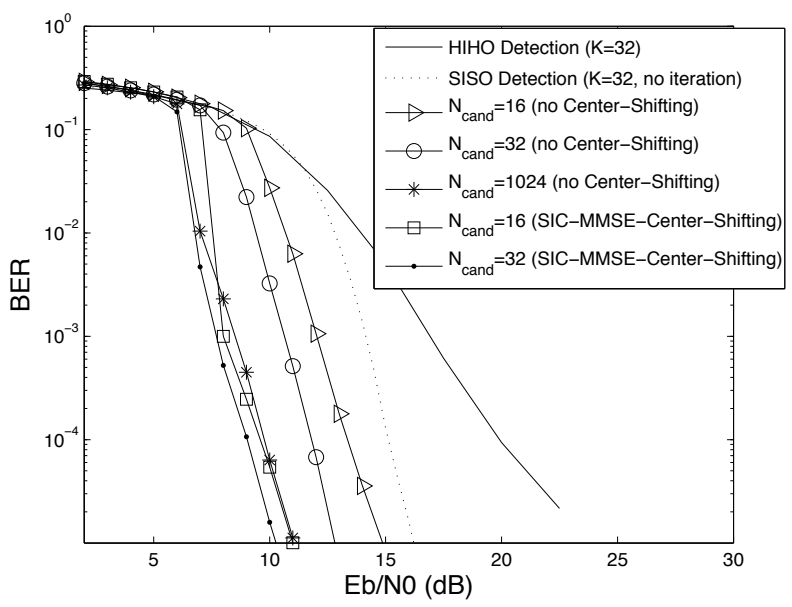

Fig. 22. Achievable BER performance of the iteratively detected half-rate RSC-coded QPSK modulated SDM system of Fig. 10 employing $(M, N)$ $=(8,4)$ AEs and the SIC-MMSE-aided iterative centre-shifting $K$-best SD receiver. (C)2008 IET, Wang, Xu, Chen, Hanzo, [49].

that even for as low as $\zeta=0.5$, the outer EXIT curve of the MC-MBER detector exhibited a good performance, which was close to that of the full-complexity MC-MBER detector of Fig. 19.

Moreover, Fig. 22 illustrates the achievable BER performance of the $K$-best SISO SD [49] in the scenario of the QPSK-modulated $(8 \times 4)$-element SDM system. In this scheme, search center $\boldsymbol{X}_{c}$ was iteratively updated with the aid of Soft-Interference-Cancellation (SIC-)MMSE [29]. Observe in Fig. 22 that the reduced-complexity $(K=16)$ centershifting SD is capable of approaching the performance of the large-search-space $(K=1024) \mathrm{SD}$ dispensing with centershifting at $\mathrm{BER}=10^{-5}$. Also, for a fixed value of $K=32$, the

BASIC SYSTEM PARAMETERS OF THE CODED SDM SCHEME OF FIG. 23.

\begin{tabular}{lr}
\hline \hline Number of transmit antennas & $M=4$ \\
Number of receive antennas & $N=3$ \\
Modulation & QPSK
\end{tabular}

Channels

Channel's coherence-time

Frequency-flat Rayleigh fading $\tau=1$ symbol duration

Detector

Interleaver blocklength

Outer channel code

Generator polynomials

Precoder

Number of inner iterations

Number of outer iterations

$$
\begin{array}{r}
\text { Max-log MAP detector } \\
200000 \text { bits } \\
\operatorname{RSC}(2,1,2) \\
\left(G_{\mathrm{r}}, G\right)=(3,2)_{8} \\
\text { URC } \\
I_{\text {in }}=1 \\
I_{\text {in }}=0-10
\end{array}
$$

iterative gain over the non-iterative receiver labeled as 'SISO detection' was doubled to approximately $6 \mathrm{~dB}$ by the SICMMSE center-shifting-aided receiver, when compared to that of iterative SD dispensing with the center-shifting. ${ }^{6}$

\section{B. Three-Stage-Concatenated Turbo SDM Systems}

Let us now consider the family of serially concatenated three-stage turbo SDM system shown in Fig. 23. More specifically, the information bits are firstly channel-encoded by the half-rate Recursive Systematic Convolutional (RSC) code and then interleaved by the first random interleaver $\Pi_{1}$ of Fig. 23. Then, the interleaved bits are further encoded by the UnityRate Convolutional (URC) code and the URC-coded bits are then interleaved by the random interleaver $\Pi_{2}$ of Fig. 23. Finally, the interleaved bits are mapped to the AEs with the aid of our SDM mapping scheme, in order to generate the space-time codewords $\boldsymbol{S}(i)$ to be transmitted to the receiver. By contrast, the receiver structure of Fig. 23 is constituted by a three-stage iterative detector, where three SISO decoders exchange their extrinsic information in the form of LLRs. Let us assume that the RSC code is used as the outer code, while considering the amalgamated combination of the URC code and the SDM mapper to be the inner code. ${ }^{7}$

Fig. 24 shows the EXIT curves of the QPSK-modulated SDM arrangements, where the corresponding SNR was varied from $0 \mathrm{~dB}$ to $10 \mathrm{~dB}$ with a step-size of $1 \mathrm{~dB}$ for generating the inner code's EXIT curves. We also plotted the outer RSC $(2,1,2)$ decoder's EXIT curve, which employed the octal generator polynomials of $(3,2)_{8}$, where the interleaver length of both interleaver $\Pi_{1}$ and $\Pi_{2}$ was set to 200000 bits. This is a sufficiently high interleaver length, which enables a good match between the EXIT-chart prediction and the MonteCarlo simulation-based BER results, as detailed in [53]. The

\footnotetext{
${ }^{6}$ For further numerical investigations as well as the complexity analysis of the SD, please refer to [48,61]. Additionally, the achievable BER performance of the SD and the MCMC can be found in [32,62].

${ }^{7}$ The benefit of this assumption is that we do not need a three-dimensional EXIT chart, whilst a three-stage concatenated scheme would require two 3D EXIT charts for visualizing the mutual information improvement benefits of exchanging extrinsic information amongst three serially concatenated SISO blocks, as detailed in [53]. This amalgamation of the above-mentioned two components is carried out by exchanging extrinsic information between these two components as many times, as necessary for achieving the highest mutual information between them and then considering them as a single SISO block.
} 
This article has been accepted for inclusion in a future issue of this journal. Content is final as presented, with the exception of pagination.

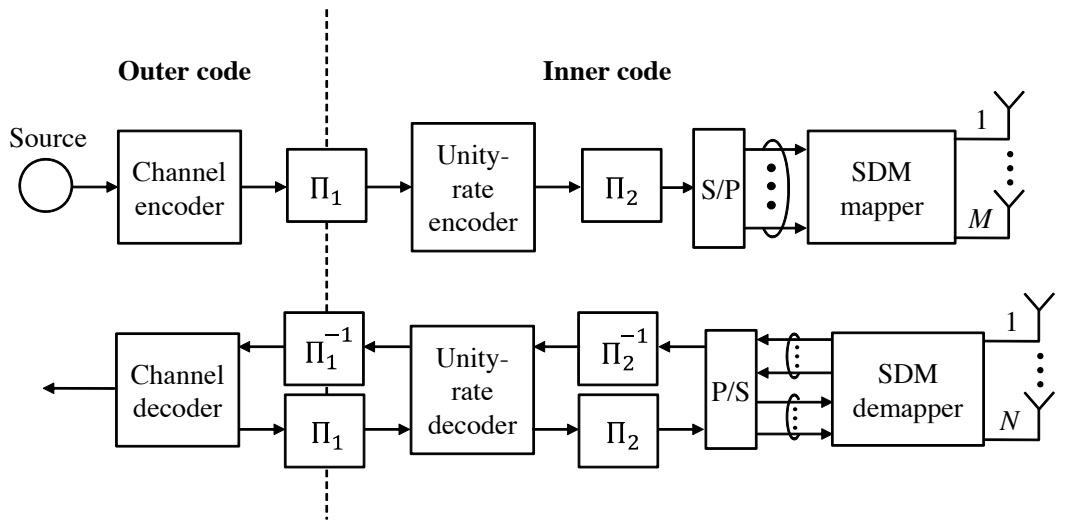

Fig. 23. Schematic of a three-stage RSC- and URC-coded SDM scheme using iterative detection.

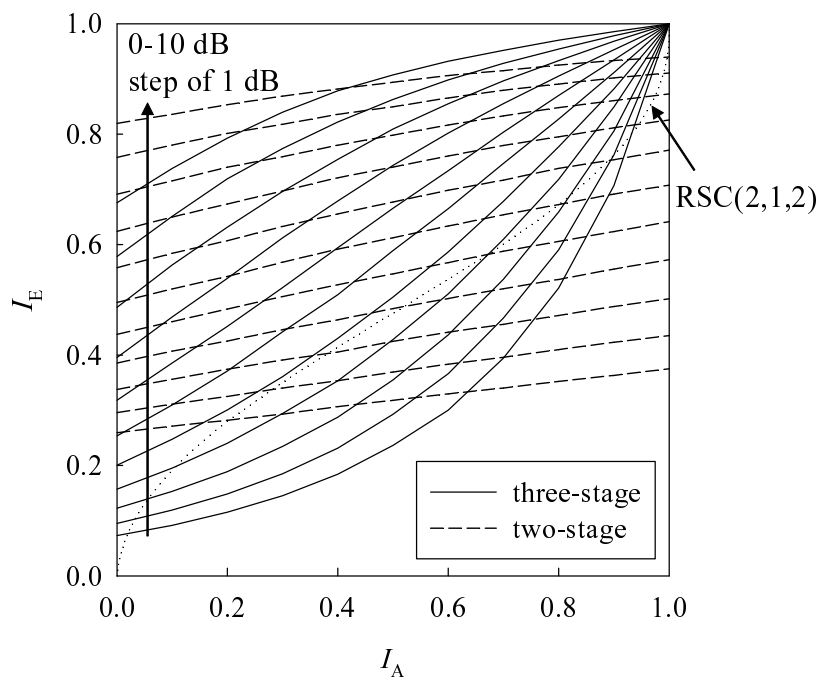

Fig. 24. EXIT chart of our RSC- and URC-coded SDM system of Fig. 23. All other system parameters were summarized in Table XI.

corresponding EXIT curves of the two-stage turbo-coded SDM system of Fig. 10 were also plotted as a benchmarker. It can be seen in Fig. 24 that the corresponding inner decoder's EXIT curves of the three-stage and two-stage systems exhibited substantially different characteristics. More specifically, the inner code's EXIT curve of the three stage system reached the point of perfect convergence to a vanishingly low BER at $\left(I_{\mathrm{A}}, I_{\mathrm{E}}\right)=(1.0,1.0)$, as the explicit benefit of employing a URC. ${ }^{8}$

Finally, Fig. 25 shows the achievable BER performance of our RSC- and URC-coded SDM system employing QPSK modulation, which had a total throughput of $R=4$ bits/symbol. The number of iterations $I$ between the outer and inner codes was varied from $I_{\text {out }}=0$ to $I_{\text {out }}=10$. As predicted from the EXIT chart of Fig. 24, the corresponding

\footnotetext{
${ }^{8}$ The role of the URC is to transform the scheme considered into an Infinite Impulse Response (IIR) arrangement, which results in an improved iterative gain by effectively spreading the extrinsic information and hence eliminating the potential error-floor.
}

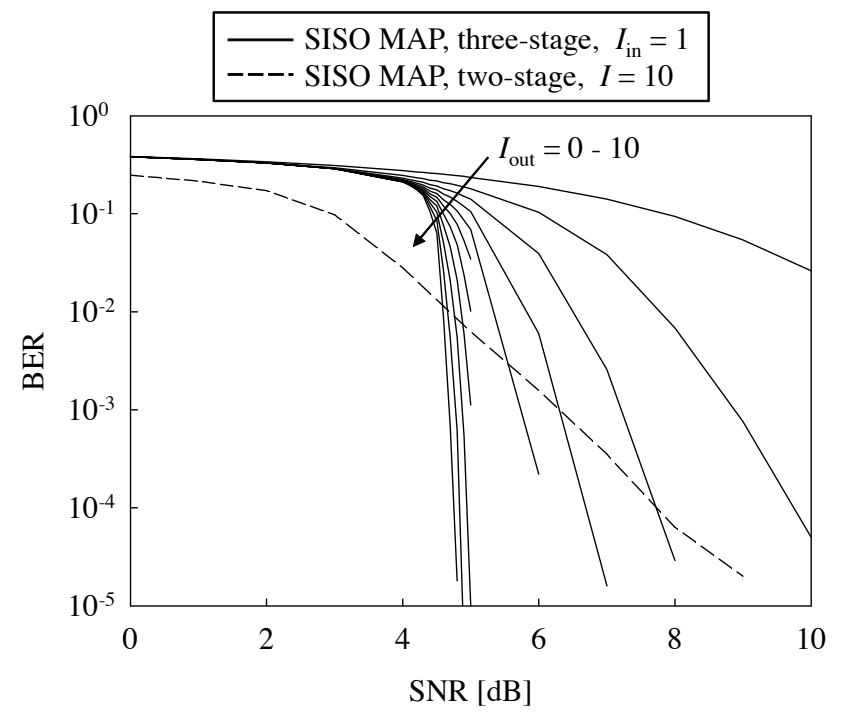

Fig. 25. Achievable BER performance of our RSC- and URC-coded SDM system of Fig. 23 employing QPSK modulation, where the number of outer iterations $I_{\text {out }}$ was changed from $I_{\text {out }}=0$ to $I_{\text {out }}=10$. All other system parameters were summarized in Table XI. The dashed-lines indicate the lowest possible SNRs, at which an infinitesimally low BER may be attained at the effective throughput of 4 bits/symbol, calculated from DCMC capacity and the maximum achievable rate.

BER curve exhibited an infinitesimally low BER at the SNR point of $4.9 \mathrm{~dB} .^{9}$

\section{Summary AND CONCLUSIONS}

In this treatise, we introduced diverse hard- and softdecision MIMO detectors in the context of SDM systems, such as the ZF, the MMSE, the MBER, the ML, the MAP,

${ }^{9}$ To elaborate a little futher, EXIT charts are useful not only for analyzing the convergence behaviour of iterative decoding, but also for designing the system architecture capable of achieving a near-capacity performance. For example, the recent turbo-coded system may be constituted by the IRregularConvolutional Codes (IRCC) [63] or the IRregular-Precoded LDCs (IRPLDC) [64], which can be optimized with the aid of the EXIT chart, so that inner- and outer-EXIT curves are matched with each other, hence having a minimal EXIT tunnel area over a wide range of SNRs. Similarly, the irregular LDPC codes [65] can also be optmized based on the EXIT chart for the sake of attaining a near-capacity performance, as shown in [66]. Furthermore, in [67] Multi-Level Bit-Interleaved Coded Modulation (ML-BICM) may be adaptively encoded with the aid of EXIT chart, where the information rate loss caused by the mismatch between channel realization and channel coding is minimized in a near-simultaneous manner. 
This article has been accepted for inclusion in a future issue of this journal. Content is final as presented, with the exception of pagination.

TABLE XII

SumMARY OF THE MMSE, THE MBER, THE ML, THE MAP, THE MCMC, THE MC-MBER DETECTORS IN THE CONTEXT OF UNCODED AND TURBO-CODED SDM SCHEMES OF FIGS. 2 AND 10, EMPLOYING THE SYSTEM PARAMETERS OF TABLES I AND X.

\begin{tabular}{|c|c|c|c|}
\hline & \multicolumn{3}{|c|}{ Linear detector } \\
\hline & $\mathbf{Z F}$ & MMSE & MBER \\
\hline Criterion & Section III-A & Section III-B & Section III-C \\
\hline Complexity & $\mathcal{O}\left(\frac{\max \left(M^{3}, N^{3}\right)}{\tau}\right)$ & $\mathcal{O}\left(\frac{\max \left(M^{3}, N^{3}\right)}{\tau}\right)$ & $\mathcal{O}\left(\frac{N_{\mathrm{g}} N_{\mathrm{b}} M^{2} N \log _{2} \mathcal{L}}{\mathcal{L}}\right)$ \\
\hline Performance $^{a}$ & $29.6 \mathrm{~dB}$ & $23.2 \mathrm{~dB}$ & $15.4 \mathrm{~dB}$ \\
\hline Criterion & $\overline{-1}$ & Section IV-B & $\overline{\text { Section IV-C }}$ \\
\hline Iterations & & $I=10$ & \\
\hline FEC rate & & 0.5 & \\
\hline Complexity & - & $\mathcal{O}\left(M^{2} N^{2}\right)$ & $\begin{array}{r}\mathcal{O}\left(N_{\mathrm{g}} N_{\mathrm{b}} M^{2}\right. \\
\left.\quad \times N \log _{2} \mathcal{L}\right)\end{array}$ \\
\hline $\begin{array}{l}\text { Performance }^{b} \\
(2 \times 2) \\
(4 \times 2) \\
(6 \times 2) \\
(8 \times 2)\end{array}$ & $\begin{array}{l}- \\
- \\
-\end{array}$ & $\begin{array}{r}2.5 \mathrm{~dB} \\
5.7 \mathrm{~dB} \\
7.7 \mathrm{~dB} \\
12.5 \mathrm{~dB}\end{array}$ & $\begin{array}{l}2.5 \mathrm{~dB} \\
5.6 \mathrm{~dB} \\
7.4 \mathrm{~dB} \\
8.8 \mathrm{~dB}\end{array}$ \\
\hline
\end{tabular}

\begin{tabular}{|c|c|c|c|c|}
\hline & \multicolumn{4}{|c|}{ Non-linear detector } \\
\hline & ML & MAP & MCMC & MC-MBER \\
\hline Criterion & Section III-D & - & Section III-E & Section III-F \\
\hline Complexity & $\mathcal{O}\left(N_{\mathrm{b}} M N\right)$ & - & $\begin{aligned} \mathcal{O}( & N_{\mathrm{MC}} N_{\mathrm{P}} M^{2} \\
& \left.\times N \log _{2} \mathcal{L}\right)\end{aligned}$ & $\begin{array}{r}\mathcal{O}\left(\max \left(N_{\mathrm{MC}} N_{\mathrm{P}} M \log _{2} \mathcal{L},\right.\right. \\
\left.\left.N_{\mathrm{g}} N_{\mathrm{b}}^{\prime}\right) \cdot M N\right)\end{array}$ \\
\hline Performance $^{a}$ & $11.7 \mathrm{~dB}$ & - & $12.9 \mathrm{~dB}$ & $15.4 \mathrm{~dB}$ \\
\hline Criterion & - & Section IV-A & Section IV-D & Section IV-E \\
\hline Iterations & & & $I=10$ & \\
\hline FEC rate & & & 0.5 & \\
\hline Complexity & - & $\mathcal{O}\left(N_{\mathrm{b}} M N\right)$ & $\begin{array}{r}\mathcal{O}\left(N_{\mathrm{MC}} N_{\mathrm{P}} M^{2}\right. \\
\left.\quad \times N \log _{2} \mathcal{L}\right)\end{array}$ & $\begin{array}{r}\mathcal{O}\left(\max \left((1-\gamma) N_{\mathrm{MC}} N_{\mathrm{P}}\right.\right. \\
\left.\left.N_{\mathrm{g}} N_{\mathrm{b}}^{\prime}\right) \cdot M^{2} N \log _{2} \mathcal{L}\right)\end{array}$ \\
\hline $\begin{array}{l}\text { Performance }^{b} \\
(2 \times 2) \\
(4 \times 2) \\
(6 \times 2) \\
(8 \times 2)\end{array}$ & $\begin{array}{l}- \\
- \\
-\end{array}$ & $\begin{array}{l}2.5 \mathrm{~dB} \\
5.6 \mathrm{~dB} \\
7.3 \mathrm{~dB} \\
8.7 \mathrm{~dB}\end{array}$ & $\begin{array}{l}2.5 \mathrm{~dB} \\
5.6 \mathrm{~dB} \\
7.4 \mathrm{~dB} \\
8.7 \mathrm{~dB}\end{array}$ & $\begin{array}{l}2.5 \mathrm{~dB} \\
5.6 \mathrm{~dB} \\
7.4 \mathrm{~dB} \\
8.8 \mathrm{~dB}\end{array}$ \\
\hline
\end{tabular}

${ }^{a}$ Performance is characterized by the SNR value recorded for BER $=10^{-3}$ in the $(3 \times 3)$-element BPSK-modulated uncoded SDM system of Fig. 2 .

${ }^{b}$ Performance is characterized by the SNR value required for BER $=10^{-3}$ in the $(M \times 2)$-element BPSK-modulated uncoded SDM system of Fig. 10 .

the MCMC and the MC-MBER detectors. In Section II, we provided the system overview and the theoretical capacity of the SDM scheme. Section III firstly reviewed the sub-class of hard-decision aided linear detectors, which are beneficial in slow-fading environments, since the calculated complexvalued weights can be reused during the coherence-time of the channels. More specifically, the ZF detector of Section III-A exhibited the poor BER performance because of the noise enhancement effects imposed on the channel's inverse matrix. The MMSE detector of Section III-B was designed to overcome this problem, hence outperforming the ZF detector. Furthermore, since the MBER detector of Section III-C directly minimized the BER, the MBER detector exhibited the best BER performance among the linear detectors, although at the expense of a high computational complexity. The MBER detector is also capable of supporting rank-deficient scenarios, while the ZF and the MMSE detectors typically exhibited the error floors in these scenarios. In contrast to the linear detectors, non-linear hard-decision detectors have the potential of approaching the optimal BER performance, but the detector weights have to be updated on a symbolby-symbol basis. The ML detector of Section III-D exhibited optimal performance owing to its exhaustive symbol procedure search, where the computational complexity increases exponentially upon increasing the multiplexing factor $M$. In order to reduce the ML detector's complexity, the MCMC detector of Section III-E invoked the Monte Carlo simulation with the aid of Markov chain sampling. Moreover, in Section III-F we proposed a new hybrid detector amalgamating the non-linear MCMC detector and the linear MBER detector, aiming for the MBER performance, while reducing its complexity in fastfading environments.

In Section IV we introduced soft-decision detectors for the two-stage serially concatenated SDM scheme of Fig. 10, where we advocated a turbo-coding assisted iterative MIMO receiver structure. Here, we extended the hard-decision detectors introduced in Section III to their SISO counterparts, which are the SISO-MMSE detector of Section IV-B, the SISO-MAP detector of Section IV-A, the SISO-MBER detector of Section IV-C, the SISO-MCMC detector of Section IV-D and the SISO MC-MBER detector of Section IV-E. Furthermore, in Section IV-E1 we proposed a novel complexity-reduction technique, namely a priori LLR thresholding, for the SISO MC-MBER detector, while characterizing the computational complexity imposed by each detector. In Section V, we characterized the SISO detectors' performance with the aid of EXIT chart, while 
This article has been accepted for inclusion in a future issue of this journal. Content is final as presented, with the exception of pagination.

analyzing the behaviour of the iterative decoding process. To elaborate a little further, in Table V-A we summarized the SISO detectors' performance and complexity comparison both for uncoded and coded SDM systems.

In this treatise, we have compared a range of diverse MIMO detectors in the context of SDM systems and characterized their complexity versus performance tradeoffs. Each suboptimal detector was designed for reducing the computational complexity of the ML and MAP detectors, which are optimal for uncoded and coded scenarios, respectively.

\begin{tabular}{|c|c|}
\hline & VII. GLOSSARY \\
\hline $\mathrm{ACO}$ & Ant-Colony Optimization \\
\hline AEs & Antenna Elements \\
\hline AWGN & Additive White Gaussian Noise \\
\hline BER & Bit-Error Ratio \\
\hline $\mathrm{BS}$ & Base Station \\
\hline $\mathrm{CC}$ & Convolutional Channel \\
\hline CCMC & $\begin{array}{l}\text { Continuous-input Continuous-output Memo- } \\
\text { ryless Channel }\end{array}$ \\
\hline CDMA & Code-Division Multiple Access \\
\hline CIRs & Channel Impulse Responses \\
\hline CSI & Channel State Information \\
\hline DCMC & $\begin{array}{l}\text { Discrete-input Continuous-output Memory- } \\
\text { less Channel }\end{array}$ \\
\hline EXIT & EXtrinsic Information Transfer \\
\hline FEC & Forward Error Correction \\
\hline GA & Genetic Algorithm \\
\hline LBER & Least Bit-Error Rate \\
\hline LDPC & Low-Density Parity-Check \\
\hline LLR & Log-Likelihood Ratio \\
\hline LMS & Least Mean Square \\
\hline MA & I Multiple Access Interference \\
\hline MAP & Maximum A Posteriori \\
\hline MBER & Minimum Bit-Error Rate \\
\hline MC-MBER & $\begin{array}{l}\text { Markov Chain assisted Minimum Bit-Error } \\
\text { Rate }\end{array}$ \\
\hline $\mathrm{MCMC}$ & Markov Chain Monte Carlo \\
\hline MIMO & Multiple-Input Multiple Output \\
\hline ML & Maximum Likelihood \\
\hline MMSE & Minimum Mean-Square Error \\
\hline MUD & Multi-User Detection \\
\hline PSK & Phase-Shift Keying \\
\hline PSO & Particle Swarm Optimization \\
\hline QAM & Quadrature Amplitude Modulation \\
\hline RLS & Recursive Least Square \\
\hline RSC & Recursive Systematic Convolutional \\
\hline SCG & Simplified Conjugate Gradient \\
\hline SD & Sphere Detection \\
\hline SDM & Space Division Multiplexing \\
\hline SISO & Soft-Input Soft-Output \\
\hline SNR & Signal-to-Noise Ratio \\
\hline STE & Space-Time Equalization \\
\hline URC & Unity-Rate Convolutional \\
\hline $\mathrm{ZF}$ & Zero-Forcing \\
\hline
\end{tabular}

\section{REFERENCES}

[1] L. Hanzo, L.-L. Yang, E.-L. Kuan, and K. Yen, Single and Multi-carrier DS-CDMA: Multi-user Detection, Space-time Spreading, Synchronisation, Networking, and Standards. John Wiley and IEEE Press, 2003.
[2] S. Verdú, Multiuser detection. Cambridge University Press, 1998.

[3] J. Mietzner, R. Schober, L. Lampe, W. Gerstacker, and P. Hoeher, "Multiple-antenna techniques for wireless communications - a comprehensive literature survey," IEEE Communications Surveys \& Tutorials, vol. 11, no. 2, pp. 87-105, 2009.

[4] A. Klein and P. W. Baier, "Linear unbiased data estimation in mobile radio systems applying CDMA," IEEE J. Sel. Areas Commun., vol. 11, no. 7, pp. 1058-1066, 1993.

[5] L. Hanzo, M. Münster, B. J. Choi, and T. Keller, OFDM and MC-CDMA for Broadband Multi-User Communications, WLANs and Broadcasting. John Wiley and IEEE Press, 2003.

[6] X. Wautelet, A. Dejonghe, and L. Vandendorpe, "MMSE-based fractional turbo receiver for space-time BICM over frequency-selective MIMO fading channels," IEEE Trans. Signal Process. , vol. 52, no. 6, pp. 1804-1809, 2004.

[7] S. Chen, A. K. Samingan, B. Mulgrew, and L. Hanzo, "Adaptive minimum-BER linear multiuser detection for DS-CDMA signals in multipath channels," IEEE Trans. Signal Process. , vol. 49, no. 6, pp. 1240-1247, 2001.

[8] S. Chen, L. Hanzo, and A. Livingstone, "MBER space-time decision feedback equalization assisted multiuser detection for multiple antenna aided SDMA systems," IEEE Trans. Signal Process., vol. 54, no. 8, pp. 3090-3098, 2006

[9] S. Chen, A. Livingstone, and L. Hanzo, "Minimum bit-error rate design for space-time equalization-based multiuser detection," IEEE Trans. Commun., vol. 54, no. 5, pp. 824-832, 2006.

[10] J. Li, G. Wei, and F. Chen, "On minimum-BER linear multiuser detection for DS-CDMA channels," IEEE Trans. Signal Process. , vol. 55 , no. 3, pp. 1093-1103, 2007.

[11] R. T. Compton, Adaptive Antennas: Concepts and Performance. Prentice Hall, 1988.

[12] S. Chen, N. N. Ahmad, and L. Hanzo, "Adaptive minimum bit-error rate beamforming," IEEE Trans. Wireless Commun. , vol. 4, no. 2, pp. 341-348, 2005.

[13] O. Damen, A. Chkeif, and J. C. Belfiore, "Lattice code decoder for space-time codes," IEEE Commun. Lett., vol. 4, no. 5, pp. 161-163, 2000.

[14] M. Juntti, T. Schlosser, and J. Lilleberg, "Genetic algorithms for multiuser detection in synchronous CDMA," in IEEE International Symposium on Information Theory, 29 1997, p. 492.

[15] C. Ergun and K. Hacioglu, "Multiuser detection using a genetic algorithm in CDMA communications systems," IEEE Trans. Commun., vol. 48, no. 8, pp. 1374-1383, 2000.

[16] K. Yen and L. Hanzo, "Genetic algorithm assited joint multiuser symbol detection and fading channel estimation for sysnchronous CDMA systems," IEEE J. Sel. Areas Commun. , vol. 19, no. 6, pp. 985-998, 2001.

[17] M. Dorigo and T. Stützle, Ant Colony Optimization. MIT Press, 2004.

[18] S. Hijazi and B. Natarajan, "Novel low-complexity DS-CDMA multiuser detector based on ant colony optimization," in IEEE 60th Vehicular Technology Conference, vol. 3, 26-29 2004, pp. 1939-1943.

[19] C. Xu, B. Hu, L. Yang, and L. Hanzo, "Ant-colony-based multiuser detection for multifunctional-antenna-array-assisted MC DS-CDMA systems," IEEE Trans. Veh. Technol. , vol. 57, no. 1, pp. 658-663, 2008.

[20] S. Yang, M. Wang, and L. Jiao, "A quantum particle swarm optimization," in IEEE Congress on Evolutionary Computation, vol. 1, 2004, pp. 320-324.

[21] Y. Zhao and J. Zheng, "Particle swarm optimization algorithm in signal detection and blind extraction," in International Symposium on Parallel Architectures, Algorithms and Networks, 10-12 2004, pp. 37-41.

[22] K. K. Soo, Y. M. Siu, W. S. Chan, L. Yang, and R. S. Chen, "Particleswarm-optimization-based multiuser detector for CDMA communications," IEEE Trans. Veh. Technol. , vol. 56, no. 5, pp. 3006-3013, 2007.

[23] Z. Yang, B. Lu, and X. Wang, "Bayesian Monte Carlo multiuser receiver for space-time coded multicarrier CDMA systems," IEEE J. Sel. Areas Commun., vol. 19, no. 8, pp. 1625-1637, 2001.

[24] R. Chen, J. S. Liu, and X. Wang, "Convergence analyses and comparisons of Markov chain Monte Carlo algorithms in digital communications," IEEE Trans. Signal Proc., vol. 50, no. 2, pp. 255-270, 2002.

[25] A. Doucet and X. Wang, "Monte Carlo methods for signal processing: a review in the statistical signal processing context," IEEE Signal Proc. Mag., vol. 22, no. 6, pp. 152-170, 2005.

[26] C. Berrou, A. Glavieux, and P. Thitimajshima, "Near Shannon limit error-correcting coding and decoding: Turbo-codes," in IEEE International Conference on Communications, vol. 2, Geneva, Switzerland, 1993, pp. 1064-1070. 
This article has been accepted for inclusion in a future issue of this journal. Content is final as presented, with the exception of pagination.

[27] L. Hanzo, T. H. Liew, and B. L. Yeap, Turbo Coding, Turbo Equalisation, and Space-Time Coding for Transmission over Fading Channels. John Wiley and IEEE Press, 2002.

[28] X. Wang and H. V. Poor, "Iterative (turbo) soft interference cancellation and decoding for coded CDMA," IEEE Trans. Commun. , vol. 47, no. 7, pp. 1046-1061, 1999.

[29] M. Tüchler, A. C. Singer, and R. Koetter, "Minimum mean squared error equalization using a priori information," IEEE Trans. Signal Process., vol. 50, no. 3, pp. 673-683, 2002.

[30] S. Tan, S. Chen, and L. Hanzo, "On multi-user EXIT chart analysis aided turbo-detected MBER beamformer designs," IEEE Trans. Wireless Commun., vol. 7, no. 1, pp. 314-323, 2008.

[31] S. Tan, J. Wang, S. X. Ng, S. Chen, and L. Hanzo, "Three-Stage Turbo MBER Multiuser Beamforming Receiver Using Irregular Convolutional Codes," IEEE Trans. Veh. Technol. , vol. 57, no. 3, pp. 1657-1663, 2008.

[32] B. Farhang-Boroujeny, H. Zhu, and Z. Shi, "Markov chain Monte Carlo algorithms for CDMA and MIMO communication systems," IEEE Trans. Signal Process., vol. 54, no. 5, pp. 1896-1909, 2006.

[33] C. Xu, R. Maunder, L.-L. Yang, and L. Hanzo, "Near-optimum multiuser detectors using soft-output ant-colony-optimization for the DS-CDMA uplink," IEEE Signal Process. Lett., pp. 137-140, 2009.

[34] B. Hochwald and S. Ten Brink, "Achieving near-capacity on a multipleantenna channel," IEEE Trans. Commun. , vol. 51, no. 3, pp. 389-399, 2003.

[35] H. Vikalo, B. Hassibi, and T. Kailath, "Iterative decoding for MIMO channels via modified sphere decoding," IEEE Trans. Wireless Commun., vol. 3, no. 6, pp. 2299-2311, 2004.

[36] V. Pauli, L. Lampe, and R. Schober, “"Turbo DPSK” using soft multiplesymbol differential sphere decoding," IEEE Trans. Inf. Theory, vol. 52, no. 4, pp. 1385-1398, 2006.

[37] S. Sugiura, S. Chen, and L. Hanzo, "Reduced-complexity iterative Markov chain MBER detection for MIMO systems," IEEE Signal Process. Lett., vol. 16, no. 3, pp. 160-163, March 2009

[38] S. Sugiura, N. Wu, and L. Hanzo, "Improved Markov chain MBER detection for steered linear dispersion coded MIMO systems," in IEEE 69th Vehicular Technology Conference (VTC2009-Spring), Barcelona, Spain, April 2009, pp. 1-5.

[39] I. E. Telatar, "Capacity of multi-antenna Gaussian channels," European transactions on telecommunications, vol. 10, no. 6, pp. 585-595, 1999.

[40] S. X. Ng and L. Hanzo, "On the MIMO channel capacity of multidimensional signal sets," IEEE Trans. Veh. Technol., vol. 55, no. 2, pp. 528-536, 2006.

[41] S. L. Campbell and C. D. Meyer, Generalized inverses of linear transformations, ser. Surveys and Reference Works in Mathematics. Pitman London, 1979.

[42] S. Chen, A. Samingan, B. Mulgrew, and L. Hanzo, "Adaptive minimumber linear multiuser detection for ds-cdma signals in multipath channels," IEEE Trans. Signal Process., vol. 49, no. 6, pp. 1240-1247, 2001.

[43] M. Alias, S. Chen, and L. Hanzo, "Multiple-antenna-aided OFDM employing genetic-algorithm-assisted minimum bit error rate multiuser detection," IEEE Trans. Veh. Technol. , vol. 54, no. 5, pp. 1713-1721, 2005.

[44] W. Yao, S. Chen, S. Tan, and L. Hanzo, "Minimum bit error rate multiuser transmission designs using particle swarm optimisation," IEEE Trans. Wireless Commun., vol. 8, no. 10, pp. 5012-5017, 2009.

[45] M. S. Bazaraa, H. D. Sherali, and C. M. Shetty, Nonlinear programming: theory and algorithms. Wiley-Interscience, 2006.

[46] S. Chen, L. Hanzo, N. Ahmad, and A. Wolfgang, "Adaptive minimum bit error rate beamforming assisted qpsk receiver," in IEEE International Conference on Communications, vol. 6, no. 6, 20-24 2004, pp. 3389 3393.

[47] S. Chen, L. Hanzo, and B. Mulgrew, "Adaptive minimum symbolerror-rate decision feedback equalization for multilevel pulse-amplitude modulation," IEEE Trans. Signal Process. , vol. 52, no. 7, pp. 2092 2101, 2004.

[48] J. Akhtman, A. Wolfgang, S. Chen, and L. Hanzo, "An optimizedhierarchy-aided approximate Log-MAP detector for MIMO systems," IEEE Trans. Wireless Commun., vol. 6, no. 5, pp. 1900-1909, 2007.

[49] L. Wang, L. Xu, S. Chen, and L. Hanzo, "Generic iterative searchcentre-shifting $K$-best sphere detection for rank-deficient SDM-OFDM systems," Electronics Letters, vol. 44, no. 8, pp. 552-553, 2008.

[50] R. Zhang and L. Hanzo, "A unified treatment of superposition coding aided communications: Theory and practice," IEEE Communications Surveys \& Tutorials, pp. 1-18, in press.

[51] S. ten Brink, "Convergence behavior of iteratively decoded parallel concatenated codes," IEEE Trans. Commun. , vol. 49, no. 10, pp. 17271737,2001
[52] N. Bonello, S. Chen, and L. Hanzo, "Low-density parity-check codes and their rateless relatives," IEEE Communications Surveys \& Tutorials, vol. 13, no. 1, pp. 3-26, 2011.

[53] L. Hanzo, O. Alamri, M. El-Hajjar, and N. Wu, Near-capacity Multifunctional MIMO Systems: Sphere-packing, Iterative Detection and Cooperation. John Wiley and IEEE Press, 2009.

[54] P. Robertson, P. Hoeher, and E. Villebrun, "Optimal and sub-optimal maximum a posteriori algorithms suitable for turbo decoding," European Transactions on Telecommunications, vol. 8, no. 2, pp. 119-125, 1997.

[55] S. Tan, S. Chen, and L. Hanzo, "MBER turbo multiuser beamforming aided QPSK receiver design using EXIT chart analysis," IEEE Veh. Technology Conference, pp. 561-565, 2007.

[56] _ - "Iterative multiuser minimum symbol error rate beamforming aided qam receiver," IEEE Signal Process. Lett., vol. 15, pp. 301 -304, 2008.

[57] S. A. Laraway and B. Farhang-Boroujeny, "Implementation of a Markov chain Monte Carlo based multiuser/MIMO detector," IEEE Trans. Circuits Syst. I: Reg. Papers, vol. 56, no. 1, pp. 246-255, 2009.

[58] S. ten Brink, "Convergence of iterative decoding," Electronics Letters, vol. 35 , no. 10, pp. 806-808, 1999 .

[59] F. Brannstrom, L. K. Rasmussen, and A. J. Grant, "Convergence analysis and optimal scheduling for multiple concatenated codes," IEEE Trans. Inf. Theory, vol. 51, no. 9, pp. 3354-3364, 2005.

[60] S. Sugiura, S. Chen, and L. Hanzo, "Markov chain minimum bit error rate detection for multi-functional MIMO systems," in IEEE International Conference on Communications, Dresden, Germany, June 2009, pp. $1-5$.

[61] L. Hanzo, J. Akhtman, L. Wang, and M. Jiang, MIMO-OFDM for LTE, WiFi and WiMAX: coherent versus non-coherent and cooperative turbotransceivers. John Wiley and IEEE Press, 2010.

[62] H. Zhu, B. Farhang-Boroujeny, and R. Chen, "On performance of sphere decoding and Markov chain Monte Carlo detection methods," in IEEE 6th Workshop on Signal Processing Advances in Wireless Communications, 2005, pp. 86-90.

[63] M. Tüchler, "Design of serially concatenated systems depending on the block length," IEEE Trans. Commun. , vol. 52, no. 2, pp. 209-218, 2004.

[64] N. Wu and L. Hanzo, "Near-capacity irregular-convolutional-codingaided irregular precoded linear dispersion codes," IEEE Trans. Veh. Technol., vol. 58, no. 6, pp. 2863-2871, 2009.

[65] T. Richardson and R. Urbanke, "The capacity of low-density paritycheck codes under message-passing decoding," IEEE Trans. Inf. Theory, vol. 47, no. 2, pp. 599-618, 2002.

[66] M. Ardakani and F. Kschischang, "A more accurate one-dimensional analysis and design of irregular LDPC codes," IEEE Trans. Commun., vol. 52, no. 12, pp. 2106-2114, 2004.

[67] S. Ibi, T. Matsumoto, R. Thoma, S. Sampei, and N. Morinaga, "EXIT chart-aided adaptive coding for multilevel BICM with turbo equalization in frequency-selective MIMO channels," IEEE Trans. Veh. Technol., vol. 56, no. 6, pp. 3757-3769, 2007.

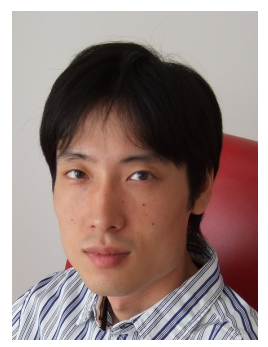

Shinya Sugiura (M'06) received the B.S. and M.S. degrees from Kyoto University, Kyoto, Japan, in 2002 and 2004, respectively, and the Ph.D. degree from University of Southampton, Southampton, UK, in 2010. Since 2004, he has been with Toyota Central R\&D Laboratories, Inc., Japan. His research has covered a range of areas in communications, including space-time modulation/demodulation, turbo coding, cooperative communications, multiuser detection, automotive antenna design as well as vehicular ad hoc networking.

Dr. Sugiura has published over 34 research papers in various journals and conference proceedings. He was awarded IEEE AP-S Japan Chapter Young Engineer Award in December 2008. 
This article has been accepted for inclusion in a future issue of this journal. Content is final as presented, with the exception of pagination.

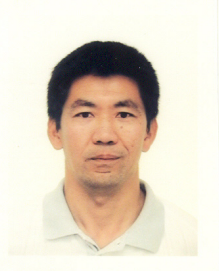

Sheng Chen (M'90-SM'97-F'08) obtained a BEng degree from the East China Petroleum Institute, Dongying, China, in 1982, and a $\mathrm{PhD}$ degree from the City University, London, in 1986, both in control engineering. In 2005, he was awarded DSc from the University of Southampton, Southampton, UK. Since 1999 he has been with the School of Electronics and Computer Science, the University of Southampton, UK. He previously held research and academic appointments at the Universities of Sheffield, Edinburgh and Portsmouth, all in UK. Professor Chen's recent research works include adaptive signal processing, wireless communications, modelling and identification of nonlinear systems, neural network and machine learning, finite-precision digital controller design, evolutionary computation methods, and optimization. He has published over 280 research papers. In the database of the world's most highly cited researchers in various disciplines, compiled by Institute for Scientific Information (ISI) of the USA, Prof. Chen is on the list of the highly cited researchers in the engineering category, see http://www.ISIHighlyCited.com.

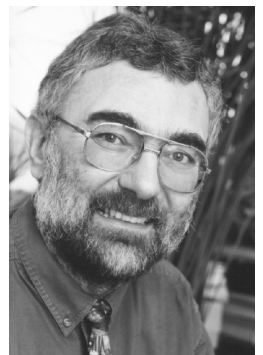

Lajos Hanzo (M'91-SM'92-F'04) FREng, FIEEE, FIET, received his degree in electronics in 1976 and his doctorate in 1983. In 2004 he was awarded the Doctor of Sciences (DSC) degree by the University of Southampton and in 2009 the honorary doctorate "Doctor Honaris Causa" by the Technical University of Budapest. During his 35-year career in telecommunications he has held various research and academic posts in Hungary, Germany and the UK. Since 1986 he has been with the School of Electronics and Computer Science, University of Southampton, UK, where he holds the chair in telecommunications. He has co-authored 20 John Wiley - IEEE Press books on mobile radio communications totalling in excess of 10000 pages, published in excess of 1000 research papers and book chapters at IEEE Xplore, acted as TPC Chair of IEEE conferences, presented keynote lectures and been awarded a number of distinctions. Currently he is directing an academic research team, working on a range of research projects in the field of wireless multimedia communications sponsored by industry, the Engineering and Physical Sciences Research Council (EPSRC) UK, the European IST Programme and the Mobile Virtual Centre of Excellence (VCE), $\mathrm{UK} . \mathrm{He}$ is an enthusiastic supporter of industrial and academic liaison and he offers a range of industrial courses. He is also an IEEE Distinguished Lecturer as well as a Governor of both the IEEE VTS. He is the Editor-in-Chief of the IEEE Press and a Chaired Prof. also at Tsinghua University, Beijing. For further information on research in progress and associated publications please refer to http://www-mobile.ecs.soton.ac.uk 